Supporting information

\title{
Beneficent and Maleficent Effects of Cations on Bufadienolide Binding to $\mathrm{Na}^{+}, \mathrm{K}^{+}$-ATPase
}

Lucy Kate Ladefoged ${ }^{1,2}$, Birgit Schiøtt ${ }^{2}$, and Natalya U. Fedosova ${ }^{1 *}$.

${ }^{1}$ Department of Biomedicine, Høegh-Guldbergsgade 10, 8000 Aarhus C, Denmark.

${ }^{2}$ Department of Chemistry, Langelandsgade 140, 8000 Aarhus C, Denmark.

*Natalya Fedosova. Email: nf@biomed.au.dk 
Table S1. Effect of cations on bufalin and cinobufagin binding. The basic media always contains $3 \mathrm{mM} \mathrm{MgCl}_{2}$.

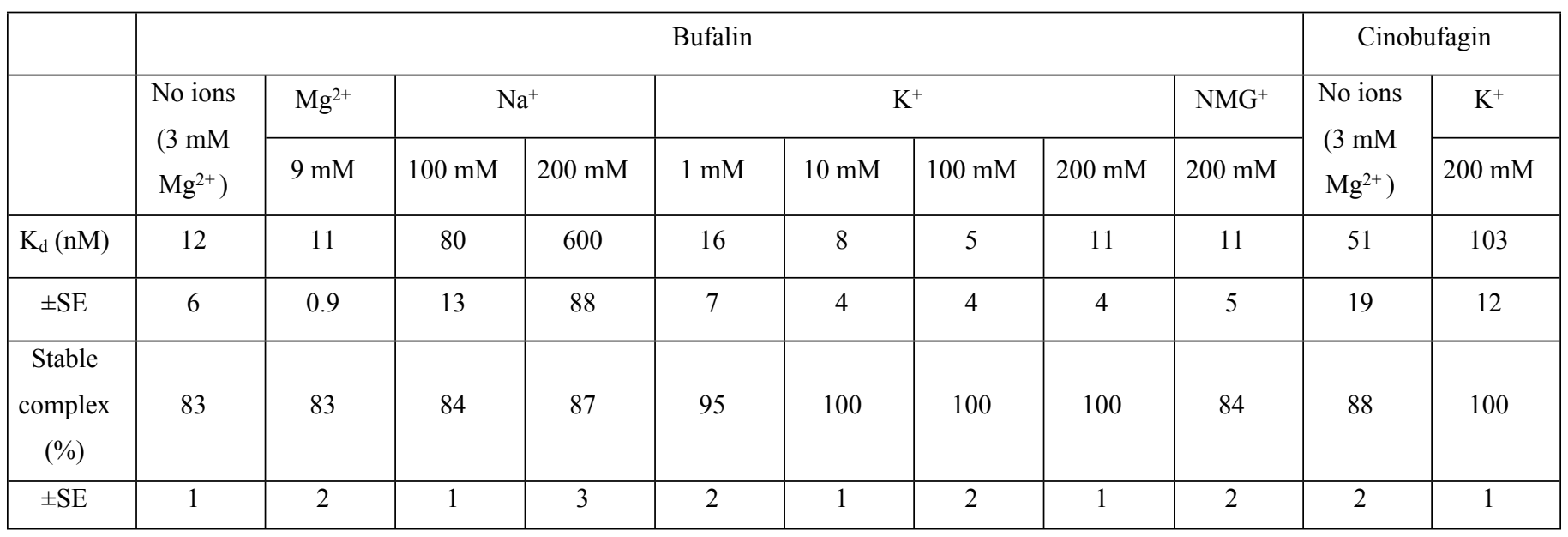


Table S2. Resulting scores of the IFD calculations of bufalin and cinobufagin into $\mathrm{Na}^{+}, \mathrm{K}^{+}$-ATPase with and without $\mathrm{K}^{+}$ions. All scores are reported as averages in $\mathrm{kcal} / \mathrm{mol}$ with the associated standard deviations (SD).

\begin{tabular}{|c|c|c|c|c|c|}
\hline \multicolumn{6}{|c|}{ Bufalin with $\mathrm{K}^{+}$} \\
\hline Cluster & $\mathrm{n}$ & Av. XP Gscore & SD & Av. IFDScore & SD \\
\hline $\mathrm{B} 1_{\mathrm{K}+}$ & 1 & -8.1 & - & -2729.6 & - \\
\hline $\mathrm{B} 2_{\mathrm{K}+}$ & 1 & -4.3 & - & -2725.6 & - \\
\hline $\mathrm{B} 3_{\mathrm{K}+}$ & 1 & -7.0 & - & -2728.2 & - \\
\hline $\mathrm{B} 4_{\mathrm{K}+}$ & 5 & -8.8 & 0.5 & -2729.9 & 0.4 \\
\hline $\mathrm{B} 5_{\mathrm{K}+}$ & 8 & -5.6 & 0.8 & -2726.8 & 0.8 \\
\hline $\mathrm{B} 6_{\mathrm{K}+}$ & 24 & -7.0 & 0.7 & -2728.6 & 0.9 \\
\hline \multicolumn{6}{|c|}{ Bufalin without $\mathrm{K}^{+}$} \\
\hline B1 & 1 & -7.4 & - & -2726.6 & - \\
\hline B2 & 1 & -4.9 & - & -2724.6 & - \\
\hline B3 & 1 & -5.4 & - & -2724.7 & - \\
\hline B4 & 10 & -7.1 & 2.1 & -2726.5 & 2.1 \\
\hline B5 & 10 & -6.2 & 0.8 & -2725.7 & 0.8 \\
\hline B6 & 15 & -7.7 & 0.8 & -2727.6 & 1.0 \\
\hline \multicolumn{6}{|c|}{ Cinobufagin with $\mathrm{K}^{+}$} \\
\hline $\mathrm{C} 1_{\mathrm{K}+}$ & 1 & -7.6 & - & -2730.9 & - \\
\hline $\mathrm{C} 2_{\mathrm{K}+}$ & 1 & -5.4 & - & -2729.1 & - \\
\hline $\mathrm{C} 3_{\mathrm{K}+}$ & 21 & -6.7 & 0.8 & -2730.5 & 1.0 \\
\hline \multicolumn{6}{|c|}{ Cinobufagin without $\mathrm{K}^{+}$} \\
\hline $\mathrm{C} 1$ & 20 & $\begin{array}{l}-6.3 \\
\end{array}$ & 0.9 & -2727.7 & 1.0 \\
\hline $\mathrm{C} 2$ & 1 & -6.4 & - & -2727.5 & - \\
\hline $\mathrm{C} 3$ & 14 & -5.7 & 0.7 & -2726.9 & 0.8 \\
\hline $\mathrm{C} 4$ & 9 & -6.1 & 1.7 & -2727.5 & 1.6 \\
\hline $\mathrm{C} 5$ & 8 & -6.7 & 1.4 & -2728.0 & 1.5 \\
\hline C6 & 18 & -6.3 & 1.3 & -2727.6 & 1.4 \\
\hline C7 & 1 & -5.2 & - & -2726.5 & - \\
\hline
\end{tabular}



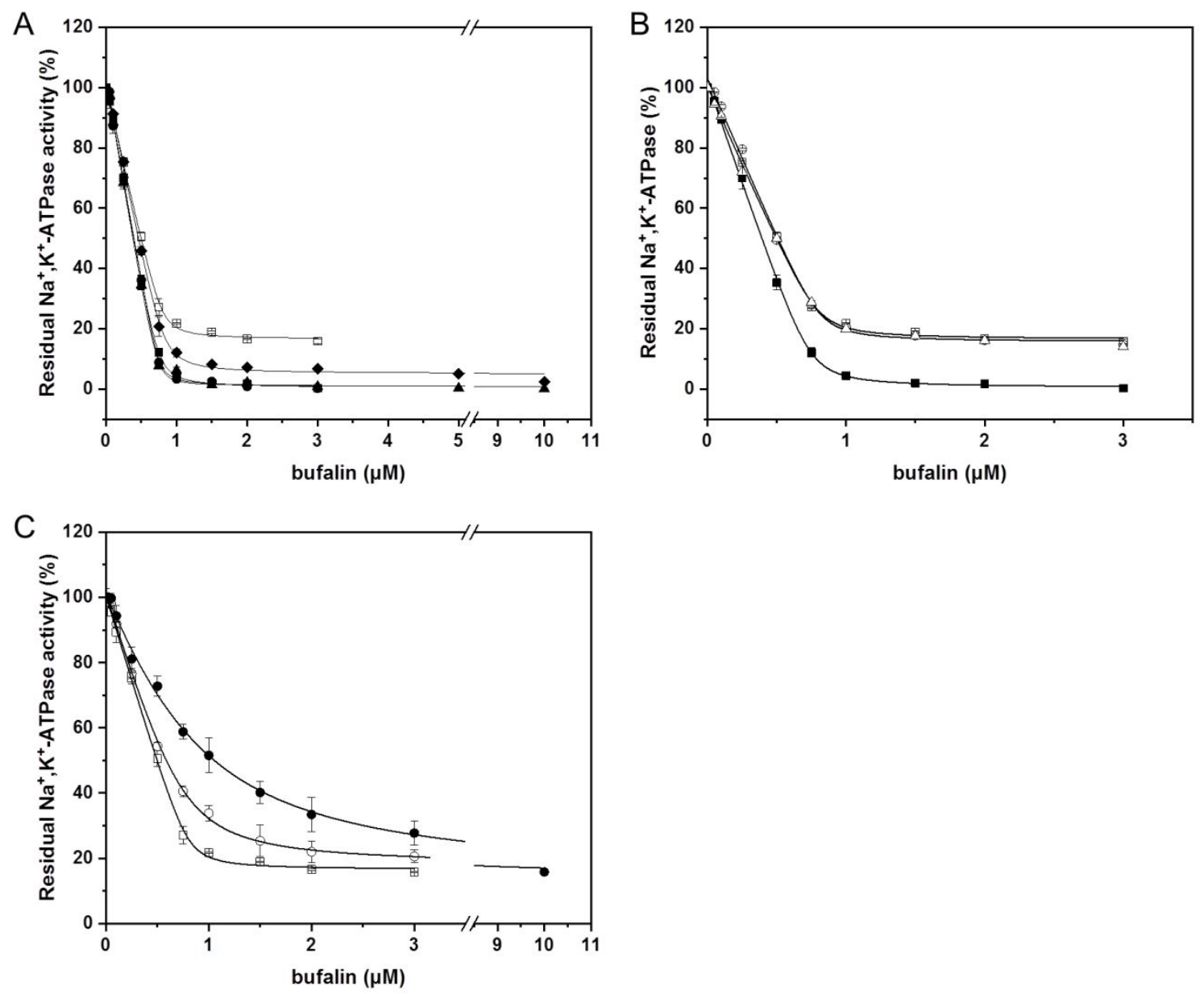

Figure S1. Cation effect on bufadienolide interactions with $\mathrm{Na}^{+}, \mathrm{K}^{+}$-ATPase as reflected by enzyme inhibition. (A) Bufalin binding in the presence of $0 \mathrm{mM} \mathrm{KCl} \mathrm{( \square ),} 1 \mathrm{mM} \mathrm{KCl}$ (•), $10 \mathrm{mM} \mathrm{KCl}(\boldsymbol{\Delta}), 100 \mathrm{mM} \mathrm{KCl}(\bullet)$, and $200 \mathrm{mM} \mathrm{KCl}(\mathbf{\bullet})$. (B) Bufalin binding in the presence of $3 \mathrm{mM} \mathrm{MgCl}_{2}(\square), 3+9 \mathrm{mM} \mathrm{MgCl}_{2}(\Delta)$ or $200 \mathrm{mM} \mathrm{NMGCl}(\circ)$, and $200 \mathrm{mM}$ $\mathrm{KCl}$ (匹). (C) Bufalin binding in the presence of $0 \mathrm{mM} \mathrm{NaCl}(\square), 100 \mathrm{mM} \mathrm{NaCl}(\circ)$, and $200 \mathrm{mM} \mathrm{NaCl}(\bullet)$. All experiments were performed in triplicates in the presence of 3 $\mathrm{mM} \mathrm{MgCl}{ }_{2}$. 

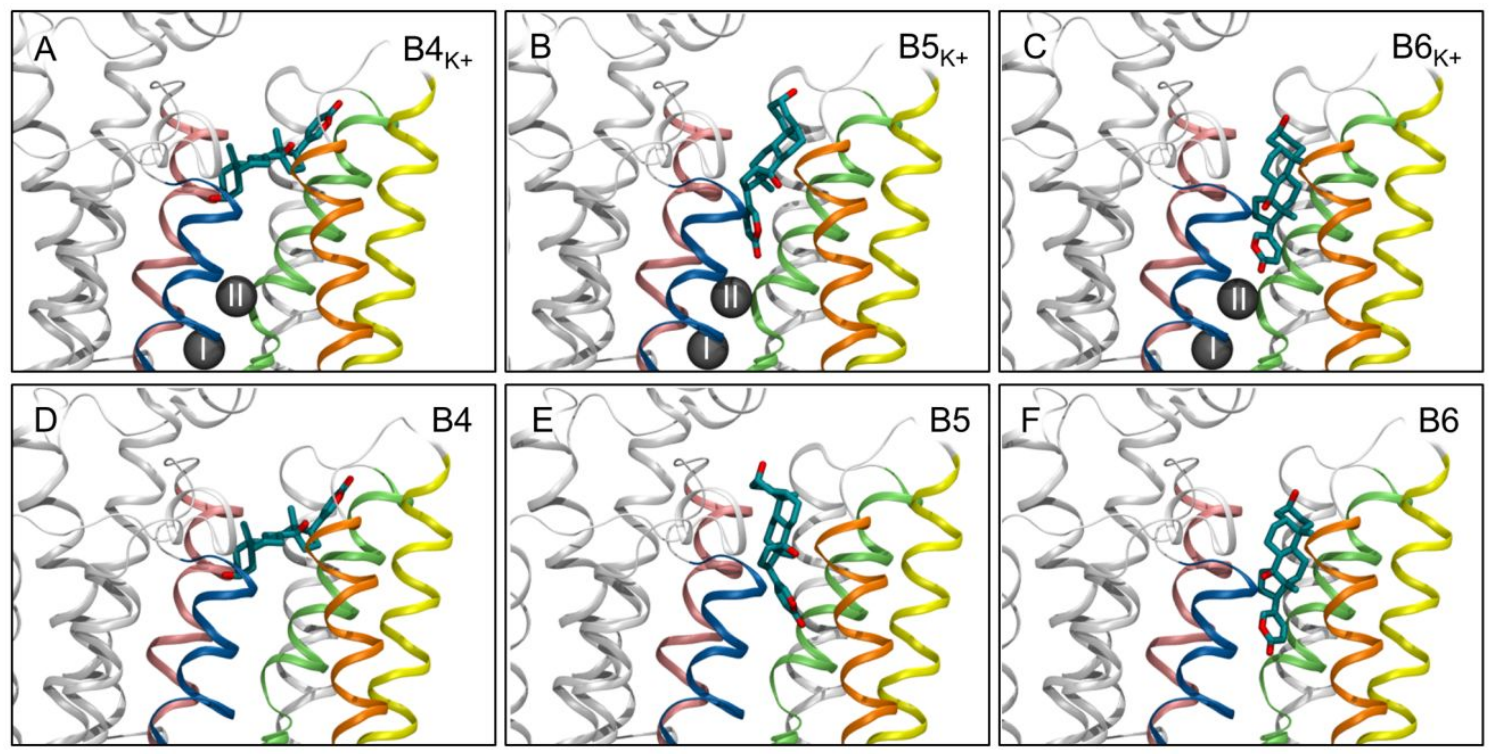

M1 M2 M3 M4 M5 M6 M7-10

Figure S2. Main binding modes of bufalin in $\mathrm{Na}^{+}, \mathrm{K}^{+}$-ATPase in the presence (ABC) or absence (DEF) of $\mathrm{K}^{+}$ions. Bufalin is shown in cyan, $\mathrm{K}^{+}$ions as gray spheres, and the enzyme as ribbons. 

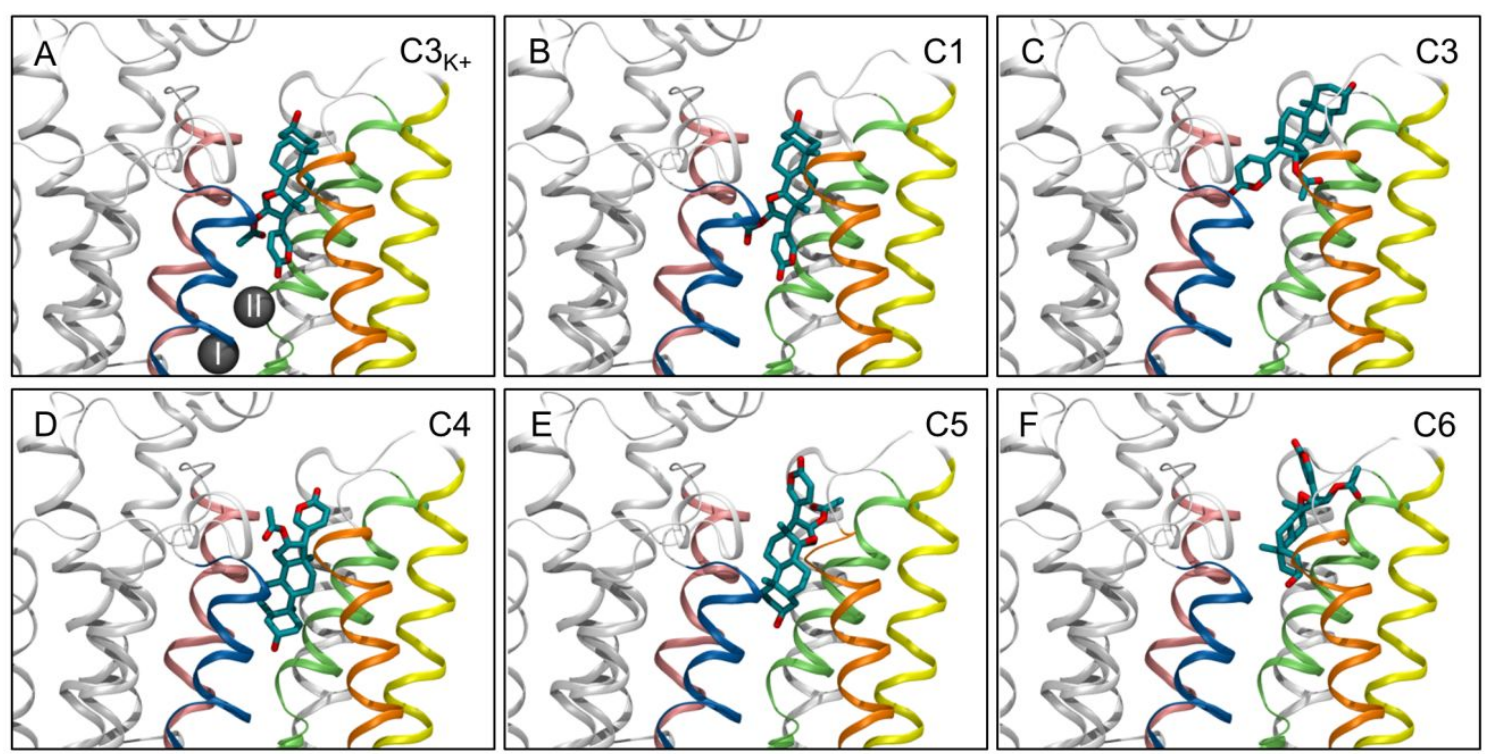

M1 M2 M3 M4 M5 M6 M7-10

Figure S3. Main binding modes of cinobufagin in $\mathrm{Na}^{+}, \mathrm{K}^{+}$-ATPase in the presence (A) or absence (BCDEF) of $\mathrm{K}^{+}$ions. Bufalin is shown in cyan, $\mathrm{K}^{+}$ions as gray spheres, and the enzyme as ribbons. 

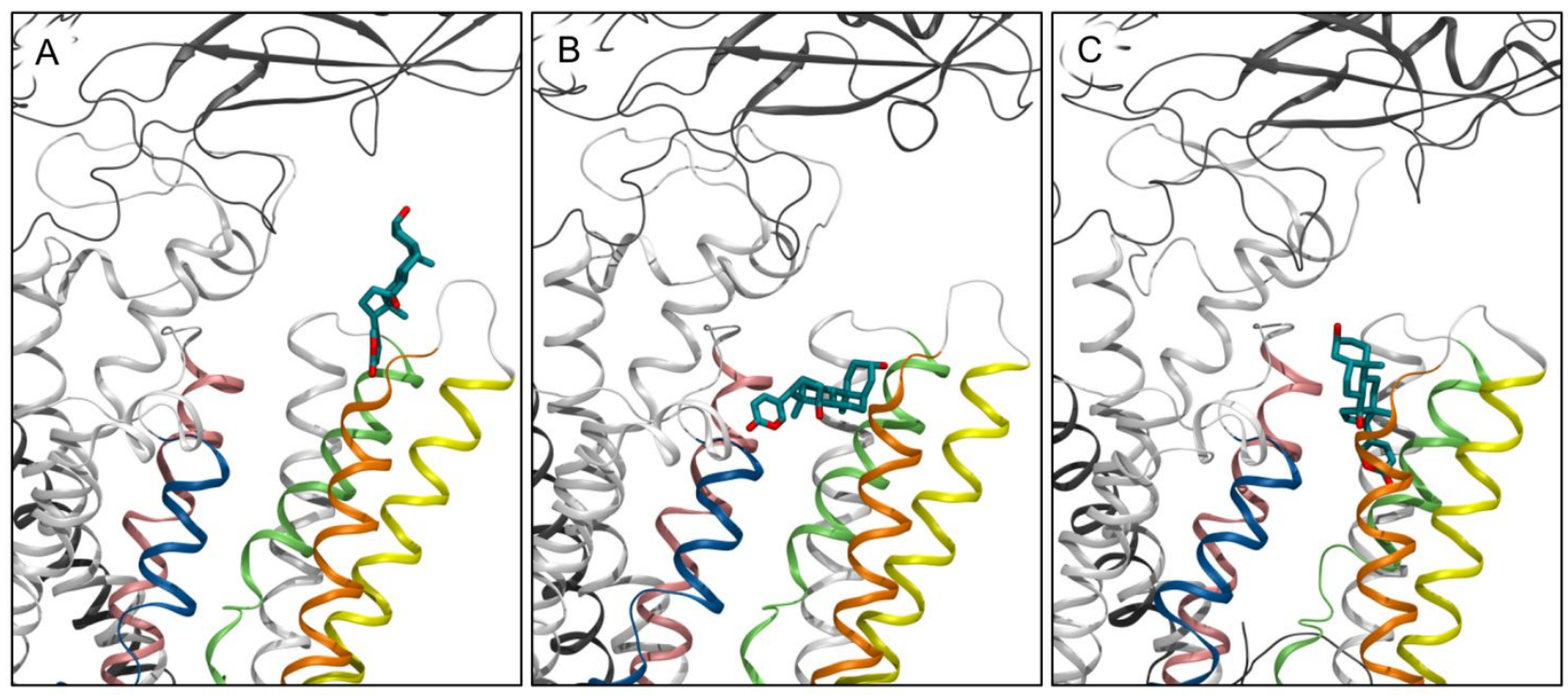

M1 M2 M3 M4 M5 M6 M7-10 $\beta$ subunit

Figure S4. Bufalin unbinding from apo $\mathrm{Na}^{+}, \mathrm{K}^{+}$-ATPase. (A) Snapshot of the most extracellular position of bufalin from MD1. (B) Snapshot of the tilted binding mode observed prior to further unbinding from $\mathrm{Na}^{+}, \mathrm{K}^{+}$-ATPase in MD1. This tilted binding mode was also observed in $\mathrm{C}_{\text {buf,K+ }}$ and $\mathrm{C}_{\text {buf }}$ from the docking calculations. (C) Snapshot of the most extracellular position of bufalin from MD2. Bufalin is shown in cyan, $\mathrm{K}^{+}$ions as gray spheres, and the enzyme as ribbons. 


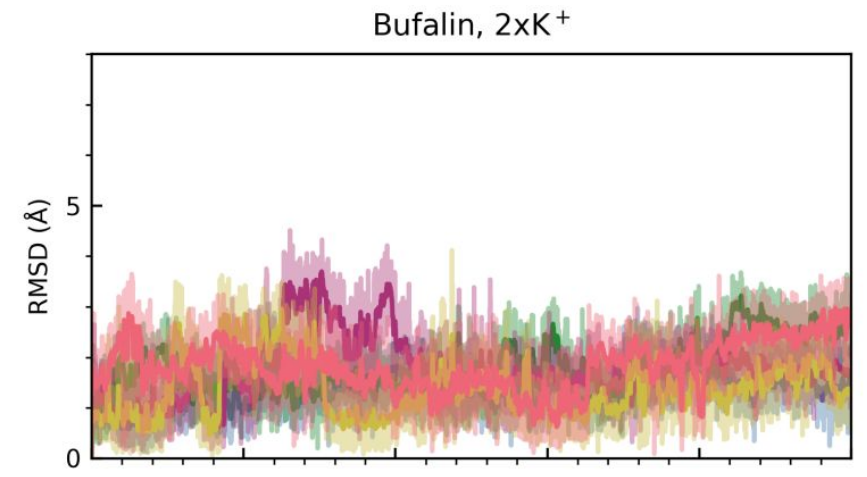

Bufalin, $1 \times \mathrm{Na}^{+}$

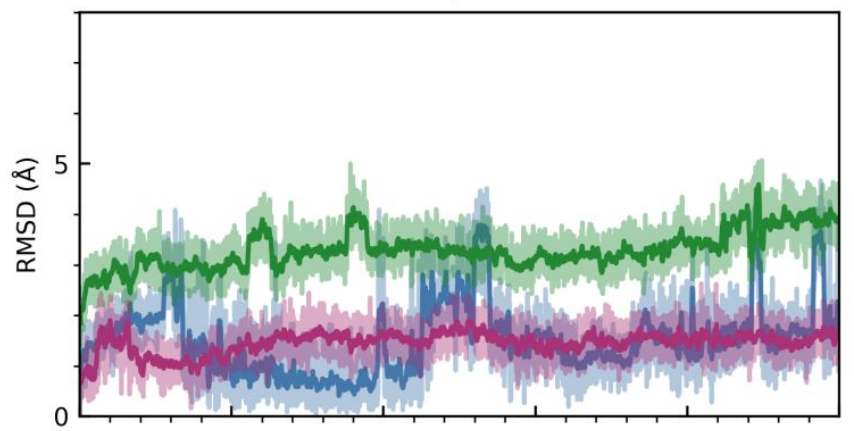

Cinobufagin, $2 \times \mathrm{K}^{+}$

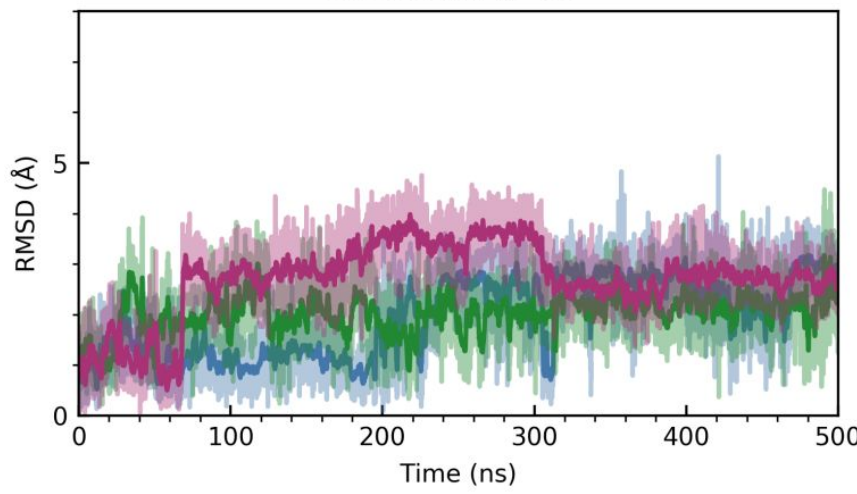

Bufalin, $2 \mathrm{xNa}^{+}$

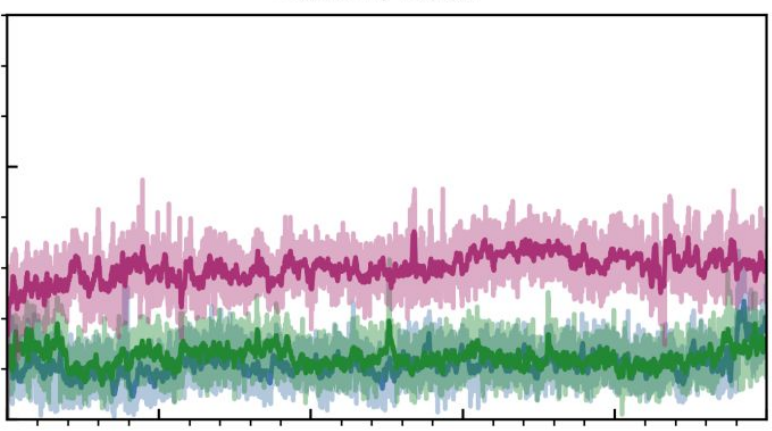

Bufalin, $1 \times \mathrm{Mg}^{2+}$

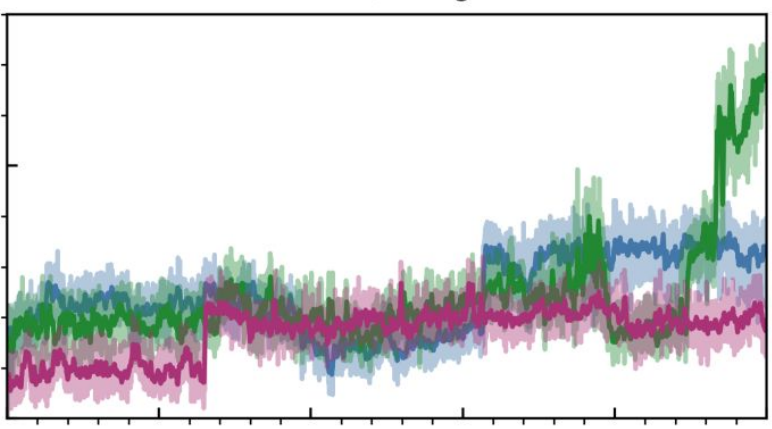

Cinobufagin, $1 \times \mathrm{Mg}^{2+}$

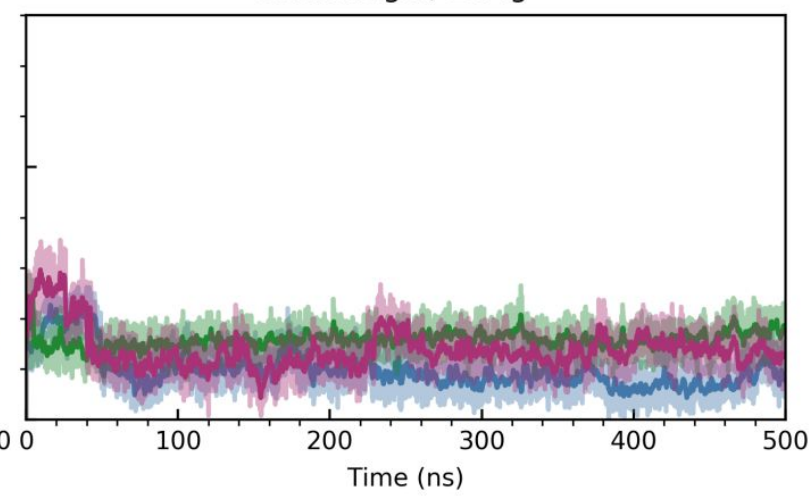

- MD1 $-\mathrm{MD2}-\mathrm{MD3}-\mathrm{MD} 4-\mathrm{MD} 5$

Figure S5. The time progressed RMSD of the ion located in cation transport site II within the enzyme. All trajectories were aligned prior to analysis using M5-10 of the enzyme. The running average is shown in opaque hues while the raw data is shown in transparent hues based on the color legend below the plot. 


\begin{tabular}{|c|c|c|c|c|c|c|c|c|c|c|c|c|}
\hline & All & M1 & M2 & M3 & M4 & M5 & M6 & 1315 & T797 & G319 & F783 & D804 \\
\hline Bufalin $2 \times K^{+}$MD1 & 2145.9 & 82.3 & 475.1 & 0.0 & 661.8 & 385.0 & 584.9 & 101.5 & 251.6 & 132.5 & 279.9 & 0.5 \\
\hline Bufalin $2 \mathrm{xK}^{+} \mathrm{MD} 2$ & 2222.7 & 71.9 & 520.6 & 0.2 & 701.7 & 607.2 & 386.9 & 112.8 & 73.2 & 127.9 & 286.6 & 45.7 \\
\hline Bufalin $2 \times K^{+}$MD3 & 2186.5 & 117.1 & 352.9 & 0.0 & 660.4 & 299.7 & 741.8 & 130.1 & 209.5 & 118.0 & 202.5 & 11.8 \\
\hline Bufalin $2 \mathrm{xK}^{+}$MD4 & 2155.5 & 82.2 & 477.7 & 0.0 & 709.0 & 489.8 & 446.9 & 103.4 & 189.8 & 133.3 & 275.1 & 15.8 \\
\hline Bufalin $2 \times K^{+}$MD5 & 2205.5 & 96.3 & 393.1 & 0.0 & 652.1 & 425.6 & 643.8 & 87.6 & 199.0 & 125.9 & 260.8 & 22.0 \\
\hline Bufalin $2 \times \mathrm{Na}^{+} \mathrm{MD} 1$ & 2387.2 & 132.4 & 468.2 & 0.0 & 472.3 & 279.7 & 335.7 & 120.0 & 132.2 & 38.1 & 162.8 & 3.6 \\
\hline Bufalin $2 \times \mathrm{Na}^{+} \mathrm{MD} 2$ & 2370.1 & 115.7 & 430.5 & 0.0 & 605.5 & 350.4 & 492.9 & 105.9 & 195.1 & 119.6 & 239.9 & 1.8 \\
\hline Bufalin $2 \times \mathrm{Na}^{+} \mathrm{MD} 3$ & 2396.1 & 61.0 & 482.4 & 0.0 & 597.1 & 346.9 & 640.2 & 93.3 & 234.2 & 114.2 & 235.9 & 7.8 \\
\hline Bufalin $1 \times \mathrm{Na}^{+} \mathrm{MD} 1$ & 2564.9 & 40.6 & 479.9 & 0.0 & 649.0 & 628.7 & 495.7 & 65.6 & 159.1 & 111.4 & 291.9 & 66.5 \\
\hline Bufalin $1 \times \mathrm{Na}^{+} \mathrm{MD} 2$ & 2480.8 & 58.4 & 444.6 & 0.0 & 789.4 & 513.8 & 402.8 & 98.3 & 158.8 & 130.3 & 290.9 & 48.8 \\
\hline Bufalin $1 \times \mathrm{Na}^{+} \mathrm{MD} 3$ & 2324.4 & 79.9 & 407.2 & 0.0 & 706.7 & 311.4 & 799.3 & 111.9 & 248.4 & 130.6 & 209.0 & 13.5 \\
\hline Bufalin $1 \times \mathrm{Mg}^{2+} \mathrm{MD} 1$ & 2227.9 & 92.6 & 441.9 & 0.0 & 632.9 & 323.9 & 696.3 & 107.1 & 234.0 & 120.7 & 211.1 & 6.0 \\
\hline Bufalin $1 \times \mathrm{Mg}^{2+} \mathrm{MD} 2$ & 2145.2 & 90.6 & 363.2 & 0.0 & 598.0 & 374.7 & 647.8 & 109.6 & 236.3 & 119.5 & 238.3 & 1.6 \\
\hline Bufalin $1 \times \mathrm{Mg}^{2+} \mathrm{MD} 3$ & 2275.7 & 83.7 & 378.9 & 0.0 & 609.5 & 339.2 & 684.3 & 107.5 & 239.0 & 123.0 & 216.4 & 1.7 \\
\hline Bufalin, no ions MD1 & 1734.8 & 94.8 & 429.0 & 0.1 & 706.7 & 396.5 & 580.1 & 104.9 & 246.1 & 135.1 & 283.7 & 1.2 \\
\hline Bufalin, no ions MD2 & 2086.2 & 74.4 & 463.9 & 0.0 & 650.3 & 360.0 & 549.6 & 93.6 & 244.4 & 130.5 & 276.5 & 0.4 \\
\hline Bufalin, no ions MD3 & 2149.6 & 67.6 & 409.0 & 0.0 & 653.3 & 344.8 & 483.3 & 115.5 & 169.2 & 123.8 & 218.8 & 15.5 \\
\hline Cinobufagin $2 \mathrm{xK}^{+} \mathrm{MD} 1$ & 2312.2 & 93.7 & 535.6 & 0.6 & 700.1 & 652.6 & 352.4 & 81.8 & 151.6 & 145.0 & 358.4 & 4.5 \\
\hline Cinobufagin $2 \mathrm{xK}^{+} \mathrm{MD} 2$ & 2366.4 & 84.5 & 518.5 & 0.5 & 742.7 & 660.9 & 368.9 & 95.3 & 153.7 & 148.8 & 362.9 & 1.6 \\
\hline Cinobufagin $2 \mathrm{xK}^{+} \mathrm{MD} 3$ & 2283.4 & 85.1 & 400.5 & 0.0 & 711.8 & 628.2 & 464.6 & 110.3 & 153.0 & 134.8 & 321.3 & 23.3 \\
\hline Cinobufagin $2 \times \mathrm{Mg}^{2+} \mathrm{MD} 1$ & 2330.2 & 87.9 & 498.2 & 0.0 & 726.8 & 422.6 & 553.7 & 143.2 & 163.6 & 126.2 & 242.1 & 9.0 \\
\hline Cinobufagin $2 \times \mathrm{Mg}^{2+} \mathrm{MD} 2$ & 2626.5 & 96.8 & 471.7 & 0.0 & 799.4 & 649.6 & 600.4 & 113.7 & 208.9 & 133.7 & 326.8 & 25.4 \\
\hline Cinobufagin $2 \times \mathrm{Mg}^{2+} \mathrm{MD} 3$ & 2430.1 & 118.7 & 436.5 & 0.0 & 753.5 & 681.7 & 401.6 & 116.6 & 102.6 & 131.1 & 336.3 & 12.8 \\
\hline
\end{tabular}

Figure S6. Enzyme/bufadienolide contacts. Each row represents a simulation repeat while each column denotes the part of the enzyme included in the contact calculation. Each cell holds the average contact value in $\AA^{2}$ for a given simulation repeat. The color of the cell relates the simulation repeat average to the overall average obtained across all molecular systems and repeats for that particular part of the enzyme. Thus, zero (light blue) on the color scale bar represents the average contact value of a given column, while green and cream represents contact values more than 3 times the standard deviation above and below the average, respectively. These extreme colors thus depict formation or disappearance of contacts compared to the average across all molecular systems i.e. a given column. 

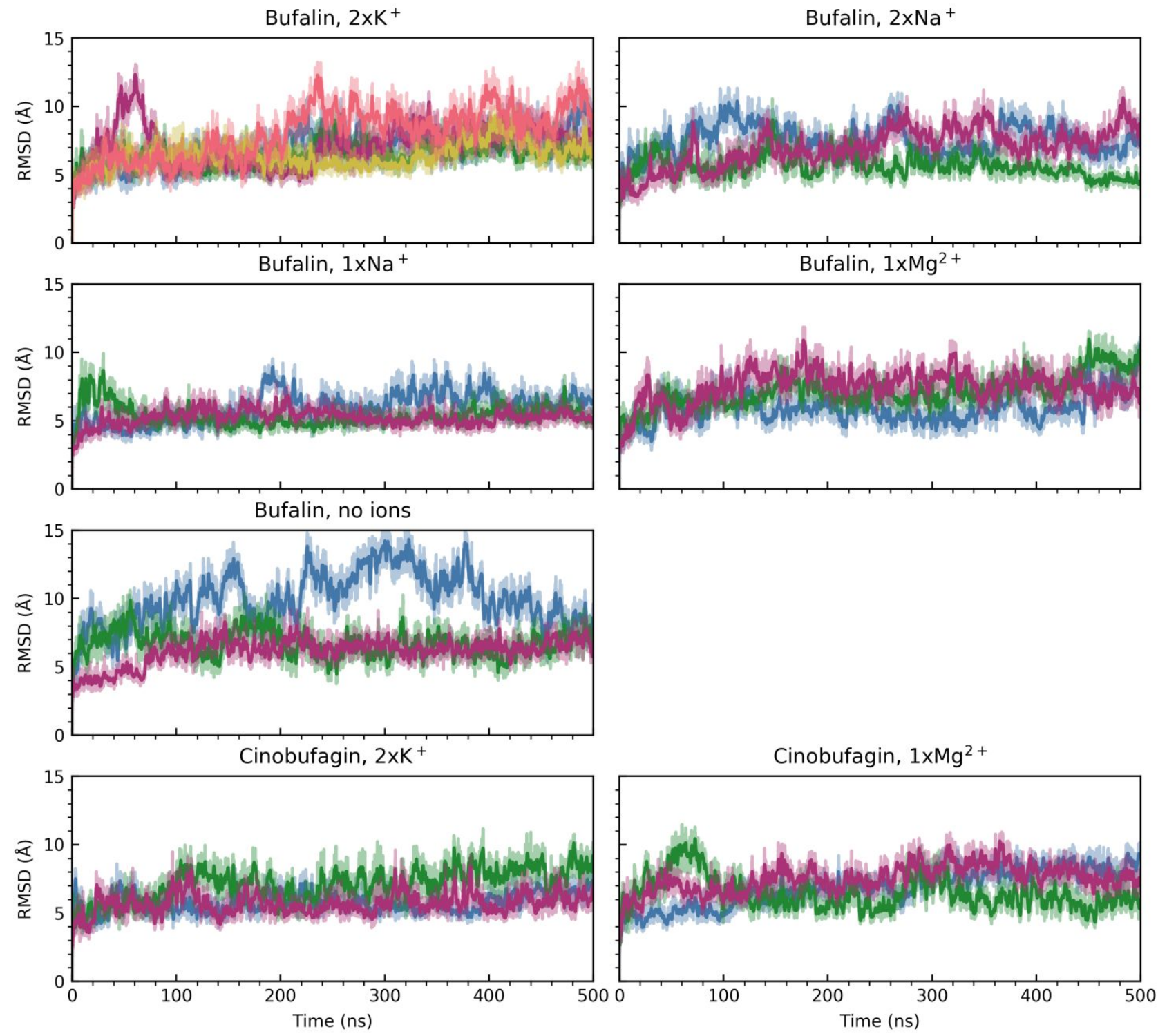

$$
-\mathrm{MD1}-\mathrm{MD2}-\mathrm{MD} 3-\mathrm{MD} 4-\mathrm{MD5}
$$

Figure S7. The time progressed RMSD of all $\mathrm{C} \alpha$ atoms of the enzyme. All trajectories were aligned prior to analysis using M5-10 of the enzyme. The running average is shown in opaque hues while the raw data is shown in transparent hues based on the color legend below the plot. 
Supporting data file S1. Bufalin itp file.

; Created by cgenff_charmm2gmx.py

[ moleculetype ]

; Name nrexcl

bufalin 3

[ atoms ]

; nr type resnr residue atom cgnr charge mass typeB chargeB massB

; residue 1 bufalin_axial rtp bufalin_axial q qsum ; charge_penalty

$1 \quad$ CG331 1 bufalin_axial $\quad$ C18 $1 ~-0.268 \quad 12.011 ; 14.448$

2 CG3RC1 1 bufalin_axial C13 $\quad 2 \quad 0.001 \quad 12.011 ; 31.241$

3 CG321 1 bufalin_axial $\quad \mathrm{C} 12 \quad 3 \quad-0.183 \quad 12.011 ; 3.363$

4 CG321 1 bufalin_axial $\quad \mathrm{C} 11 \quad 4 \quad-0.182 \quad 12.011 ; 0.000$

$5 \quad$ CG311 1 bufalin axial $\quad$ C9 $\quad 5 \quad-0.090 \quad 12.011 ; 14.705$

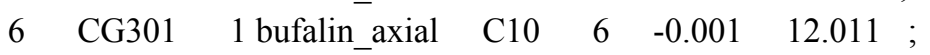

$\begin{array}{lllllll}7 & \text { CG331 } 1 \text { bufalin_axial } & \mathrm{C} 19 & 7 & -0.274 & 12.011 ;\end{array}$

$8 \quad$ CG321 1 bufalin_axial $\quad \mathrm{C} 1 \quad 8 \quad-0.180 \quad 12.011$;

9 CG321 1 bufalin_axial $\quad$ C2 $\quad 9 \quad-0.179 \quad 12.011$;

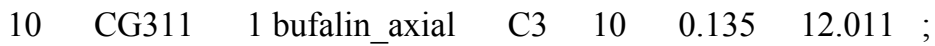

11 OG311 1 bufalin_axial O3 $11 \quad-0.650 \quad 15.999$;

12 CG321 1 bufalin_axial C4 $12 \quad-0.177 \quad 12.011$;

13 CG311 1 bufalin_axial C5 $13 \quad-0.090 \quad 12.011$;

14 CG321 1 bufalin_axial C6 $14 \quad-0.181 \quad 12.011$;

15 CG321 1 bufalin_axial C7 $15 \quad-0.181 \quad 12.011 ; 14.515$

16 CG311 1 bufalin_axial C8 $16 \quad-0.090 \quad 12.011 ; 27.227$

17 CG3RC1 1 bufalin_axial $\quad$ C14 $17 \quad 0.238 \quad 12.011 ; 23.529$

18 OG311 1 bufalin_axial O14 $18 \quad-0.651 \quad 15.999 \quad ; 21.065$

19 CG3C52 1 bufalin_axial $\quad$ C15 $19 \quad-0.181 \quad 12.011 ; 8.990$

20 CG3C52 1 bufalin_axial $\quad$ C16 $20 \quad-0.177 \quad 12.011 ; 11.730$

21 CG3C51 1 bufalin_axial $\mathrm{C} 17 \quad 21 \quad-0.039 \quad 12.011 ; 75.186$

22 CG2R62 1 bufalin_axial $\quad$ C20 $22 \quad 0.032 \quad 12.011 ; 72.103$

23 CG2R62 1 bufalin_axial $\quad$ C22 $23 \quad-0.272 \quad 12.011 ; 12.532$

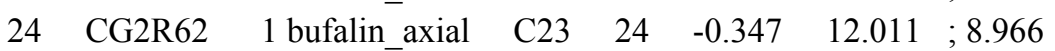

25 CG2R63 1 bufalin_axial $\quad$ C24 $25 \quad 0.490 \quad 12.011 \quad ; 2.500$

26 OG2D4 1 bufalin_axial O24 $26 \quad-0.460 \quad 15.999$;

27 OG3R60 1 bufalin_axial O21 $27 \quad-0.362 \quad 15.999 ; 59.051$

28 CG2R62 1 bufalin_axial C21 $28 \quad 0.119 \quad 12.011 ; 84.241$

29 HGA3 1 bufalin_axial H181 $29 \quad 0.090 \quad 1.008$;

30 HGA3 1 bufalin_axial H182 $30 \quad 0.090 \quad 1.008$;

31 HGA3 1 bufalin_axial H183 $31 \quad 0.090 \quad 1.008$;

32 HGA2 1 bufalin_axial H121 $32 \quad 0.090 \quad 1.008$;

33 HGA2 1 bufalin_axial H122 $33 \quad 0.090 \quad 1.008$;

34 HGA2 1 bufalin_axial H111 $34 \quad 0.090 \quad 1.008$;

35 HGA2 1 bufalin_axial H112 $35 \quad 0.090 \quad 1.008$;

36 HGA1 1 bufalin_axial H9 $36 \quad 0.090 \quad 1.008$;

$\begin{array}{llllll}37 & \text { HGA3 } & 1 \text { bufalin_axial H191 } & 37 & 0.090 & 1.008 \text {; }\end{array}$ 


\begin{tabular}{|c|c|c|c|c|c|c|c|}
\hline 38 & HGA3 & 1 bufalin_axial & H192 & 38 & 0.090 & 1.008 & \\
\hline 39 & HGA3 & 1 bufalin_axial & H193 & 39 & 0.090 & 1.008 & ; \\
\hline 40 & HGA2 & 1 bufalin_axial & H11 & 40 & 0.090 & 1.008 & ; \\
\hline 41 & HGA2 & 1 bufalin_axial & H12 & 41 & 0.090 & 1.008 & ; \\
\hline 42 & HGA2 & 1 bufalin_axial & $\mathrm{H} 21$ & 42 & 0.090 & 1.008 & . \\
\hline 43 & HGA2 & 1 bufalin_axial & $\mathrm{H} 22$ & 43 & 0.090 & 1.008 & ; \\
\hline 44 & HGA1 & 1 bufalin_axial & H3 & 44 & 0.090 & 1.008 & \\
\hline 45 & HGP1 & 1 bufalin axial & $\mathrm{HO} 3$ & 45 & 0.419 & 1.008 & ; \\
\hline 46 & HGA2 & 1 bufalin_axial & H41 & 46 & 0.090 & 1.008 & . \\
\hline 47 & HGA2 & 1 bufalin_axial & $\mathrm{H} 42$ & 47 & 0.090 & 1.008 & ; \\
\hline 48 & HGA1 & 1 bufalin_axial & H5 & 48 & 0.090 & 1.008 & \\
\hline 49 & HGA2 & 1 bufalin_axial & H61 & 49 & 0.090 & 1.008 & \\
\hline 50 & HGA2 & 1 bufalin_axial & $\mathrm{H} 62$ & 50 & 0.090 & 1.008 & ; \\
\hline 51 & HGA2 & 1 bufalin_axial & $\mathrm{H} 71$ & 51 & 0.090 & 1.008 & \\
\hline 52 & HGA2 & 1 bufalin_axial & $\mathrm{H} 72$ & 52 & 0.090 & 1.008 & \\
\hline 53 & HGA1 & 1 bufalin_axial & $\mathrm{H} 8$ & 53 & 0.090 & 1.008 & ; 2.295 \\
\hline 54 & HGP1 & 1 bufalin_axial & HO14 & 54 & 0.420 & 1.008 & ; 3.534 \\
\hline 55 & HGA2 & 1 bufalin axial & H151 & 55 & 0.090 & 1.008 & $; 0.750$ \\
\hline 56 & HGA2 & 1 bufalin_axial & H152 & 56 & 0.090 & 1.008 & $; 0.750$ \\
\hline 57 & HGA2 & 1 bufalin_axial & H161 & 57 & 0.090 & 1.008 & ; 1.500 \\
\hline 58 & HGA2 & 1 bufalin_axial & H162 & 58 & 0.090 & 1.008 & ; 1.500 \\
\hline 59 & HGA1 & 1 bufalin_axial & H17 & 59 & 0.090 & 1.008 & ; 3.841 \\
\hline 60 & HGR62 & 1 bufalin_axial & $\mathrm{H} 2$ & 60 & 0.268 & 1.008 & ; 6.142 \\
\hline 61 & HGR62 & 1 bufalin_axial & $\mathrm{H} 23$ & 61 & 0.199 & 1.008 & ; \\
\hline 62 & HGR62 & 1 bufalin axial & H1 & 62 & 0.284 & 1.008 & ; 84.726 \\
\hline
\end{tabular}

[ bonds ]

; ai aj funct $\mathrm{c} 0 \mathrm{c} 1 \mathrm{c} 2 \mathrm{c} 3$

$\begin{array}{ccc}1 & 2 & 1 \\ 1 & 29 & 1 \\ 1 & 30 & 1 \\ 1 & 31 & 1 \\ 2 & 17 & 1 \\ 2 & 3 & 1 \\ 2 & 21 & 1 \\ 3 & 33 & 1 \\ 3 & 4 & 1 \\ 3 & 32 & 1 \\ 4 & 34 & 1 \\ 4 & 5 & 1 \\ 4 & 35 & 1 \\ 5 & 36 & 1 \\ 5 & 6 & 1 \\ 5 & 16 & 1 \\ 6 & 13 & 1 \\ 6 & 7 & 1\end{array}$




$\begin{array}{ccc}6 & 8 & 1 \\ 7 & 37 & 1 \\ 7 & 39 & 1 \\ 7 & 38 & 1 \\ 8 & 9 & 1 \\ 8 & 41 & 1 \\ 8 & 40 & 1 \\ 9 & 10 & 1 \\ 9 & 43 & 1 \\ 9 & 42 & 1 \\ 10 & 44 & 1 \\ 10 & 11 & 1 \\ 10 & 12 & 1 \\ 11 & 45 & 1 \\ 12 & 13 & 1 \\ 12 & 46 & 1 \\ 12 & 47 & 1 \\ 13 & 48 & 1 \\ 13 & 14 & 1 \\ 14 & 49 & 1 \\ 14 & 50 & 1 \\ 14 & 15 & 1 \\ 15 & 51 & 1 \\ 15 & 52 & 1 \\ 15 & 16 & 1 \\ 16 & 17 & 1 \\ 16 & 53 & 1 \\ 17 & 19 & 1 \\ 17 & 18 & 1 \\ 18 & 54 & 1 \\ 19 & 20 & 1 \\ 19 & 55 & 1 \\ 19 & 56 & 1 \\ 20 & 57 & 1 \\ 20 & 58 & 1 \\ 20 & 21 & 1 \\ 21 & 59 & 1 \\ 21 & 22 & 1 \\ 22 & 28 & 1 \\ 22 & 23 & 1 \\ 23 & 60 & 1 \\ 23 & 24 & 1 \\ 24 & 25 & 1 \\ 24 & 61 & 1 \\ 25 & 26 & 1 \\ 25 & 27 & 1\end{array}$




$\begin{array}{lll}27 & 28 & 1 \\ 28 & 62 & 1\end{array}$

[pairs ]

; ai aj funct $\mathrm{c} 0 \mathrm{c} 1 \mathrm{c} 2 \mathrm{c} 3$

$\begin{array}{llll}1 & 33 & 1\end{array}$

$\begin{array}{lll}1 & 4 & 1\end{array}$

$1 \quad 16 \quad 1$

$1 \quad 18 \quad 1$

$1 \quad 19 \quad 1$

$\begin{array}{lll}1 & 20 & 1\end{array}$

$\begin{array}{lll}1 & 22 & 1\end{array}$

$\begin{array}{lll}1 & 59 & 1\end{array}$

$\begin{array}{lll}1 & 32 & 1\end{array}$

$\begin{array}{lll}2 & 34 & 1\end{array}$

$\begin{array}{lll}2 & 35 & 1\end{array}$

$\begin{array}{lll}2 & 5 & 1\end{array}$

$\begin{array}{lll}2 & 23 & 1\end{array}$

$\begin{array}{lll}2 & 15 & 1\end{array}$

$\begin{array}{lll}2 & 53 & 1\end{array}$

$\begin{array}{lll}2 & 54 & 1\end{array}$

$\begin{array}{lll}2 & 55 & 1\end{array}$

$\begin{array}{lll}2 & 56 & 1\end{array}$

$\begin{array}{lll}2 & 57 & 1\end{array}$

$\begin{array}{lll}2 & 58 & 1\end{array}$

$\begin{array}{lll}2 & 28 & 1\end{array}$

$\begin{array}{lll}3 & 36 & 1\end{array}$

$\begin{array}{lll}3 & 6 & 1\end{array}$

$\begin{array}{lll}3 & 16 & 1\end{array}$

$\begin{array}{lll}3 & 18 & 1\end{array}$

$\begin{array}{lll}3 & 19 & 1\end{array}$

$\begin{array}{lll}3 & 20 & 1\end{array}$

$\begin{array}{lll}3 & 22 & 1\end{array}$

$\begin{array}{lll}3 & 59 & 1\end{array}$

$\begin{array}{lll}3 & 29 & 1\end{array}$

$\begin{array}{lll}3 & 30 & 1\end{array}$

$\begin{array}{lll}3 & 31 & 1\end{array}$

$\begin{array}{lll}4 & 7 & 1\end{array}$

$\begin{array}{lll}4 & 8 & 1\end{array}$

$\begin{array}{lll}4 & 13 & 1\end{array}$

$\begin{array}{lll}4 & 15 & 1\end{array}$

$\begin{array}{lll}4 & 17 & 1\end{array}$

$\begin{array}{lll}4 & 21 & 1\end{array}$

$\begin{array}{lll}4 & 53 & 1\end{array}$

$\begin{array}{lll}5 & 33 & 1\end{array}$

$\begin{array}{lll}5 & 19 & 1\end{array}$ 


$\begin{array}{ccc}5 & 37 & 1 \\ 5 & 38 & 1 \\ 5 & 39 & 1 \\ 5 & 40 & 1 \\ 5 & 9 & 1 \\ 5 & 12 & 1 \\ 5 & 14 & 1 \\ 5 & 48 & 1 \\ 5 & 41 & 1 \\ 5 & 51 & 1 \\ 5 & 52 & 1 \\ 5 & 18 & 1 \\ 5 & 32 & 1 \\ 6 & 34 & 1 \\ 6 & 49 & 1 \\ 6 & 10 & 1 \\ 6 & 43 & 1 \\ 6 & 35 & 1 \\ 6 & 15 & 1 \\ 6 & 46 & 1 \\ 6 & 17 & 1 \\ 6 & 50 & 1 \\ 6 & 53 & 1 \\ 6 & 47 & 1 \\ 6 & 42 & 1 \\ 7 & 40 & 1 \\ 7 & 9 & 1 \\ 7 & 12 & 1 \\ 7 & 14 & 1 \\ 7 & 48 & 1 \\ 7 & 41 & 1 \\ 7 & 36 & 1 \\ 7 & 16 & 1 \\ 8 & 37 & 1 \\ 8 & 38 & 1 \\ 8 & 39 & 1 \\ 8 & 11 & 1 \\ 8 & 12 & 1 \\ 8 & 14 & 1 \\ 8 & 48 & 1 \\ 8 & 44 & 1 \\ 8 & 36 & 1 \\ 8 & 16 & 1 \\ 9 & 45 & 1 \\ 9 & 13 & 1 \\ 9 & 46 & 1\end{array}$




$\begin{array}{ccc}9 & 47 & 1 \\ 10 & 41 & 1 \\ 10 & 48 & 1 \\ 10 & 14 & 1 \\ 10 & 40 & 1 \\ 11 & 42 & 1 \\ 11 & 43 & 1 \\ 11 & 13 & 1 \\ 11 & 46 & 1 \\ 11 & 47 & 1 \\ 12 & 42 & 1 \\ 12 & 43 & 1 \\ 12 & 45 & 1 \\ 12 & 15 & 1 \\ 12 & 49 & 1 \\ 12 & 50 & 1 \\ 13 & 52 & 1 \\ 13 & 37 & 1 \\ 13 & 38 & 1 \\ 13 & 39 & 1 \\ 13 & 40 & 1 \\ 13 & 44 & 1 \\ 13 & 16 & 1 \\ 13 & 41 & 1 \\ 13 & 51 & 1 \\ 13 & 36 & 1 \\ 14 & 46 & 1 \\ 14 & 47 & 1 \\ 14 & 17 & 1 \\ 14 & 53 & 1 \\ 15 & 48 & 1 \\ 15 & 18 & 1 \\ 15 & 19 & 1 \\ 15 & 36 & 1 \\ 16 & 34 & 1 \\ 16 & 35 & 1 \\ 16 & 49 & 1 \\ 16 & 50 & 1 \\ 16 & 20 & 1 \\ 16 & 21 & 1 \\ 16 & 54 & 1 \\ 16 & 55 & 1 \\ 16 & 56 & 1 \\ 17 & 33 & 1 \\ 17 & 52 & 1 \\ 17 & 51 & 1 \\ & & \end{array}$




$\begin{array}{lll}17 & 36 & 1 \\ 17 & 22 & 1 \\ 17 & 57 & 1 \\ 17 & 58 & 1 \\ 17 & 59 & 1 \\ 17 & 29 & 1 \\ 17 & 30 & 1 \\ 17 & 31 & 1 \\ 17 & 32 & 1 \\ 18 & 20 & 1 \\ 18 & 53 & 1 \\ 18 & 55 & 1 \\ 18 & 56 & 1 \\ 18 & 21 & 1 \\ 19 & 53 & 1 \\ 19 & 54 & 1 \\ 19 & 59 & 1 \\ 19 & 22 & 1 \\ 20 & 23 & 1 \\ 20 & 28 & 1 \\ 21 & 33 & 1 \\ 21 & 24 & 1 \\ 21 & 62 & 1 \\ 21 & 55 & 1 \\ 21 & 56 & 1 \\ 21 & 27 & 1 \\ 21 & 60 & 1 \\ 21 & 29 & 1 \\ 21 & 30 & 1 \\ 21 & 31 & 1 \\ 21 & 32 & 1 \\ 22 & 25 & 1 \\ 22 & 57 & 1 \\ 22 & 58 & 1 \\ 22 & 61 & 1 \\ 23 & 62 & 1 \\ 23 & 26 & 1 \\ 23 & 59 & 1 \\ 23 & 27 & 1 \\ 24 & 28 & 1 \\ 25 & 60 & 1 \\ 25 & 62 & 1 \\ 26 & 28 & 1 \\ 26 & 61 & 1 \\ 27 & 61 & 1 \\ 28 & 59 & 1 \\ & & \end{array}$




$\begin{array}{clll}28 & 60 & 1 \\ 32 & 34 & 1 \\ 32 & 35 & 1 \\ 33 & 34 & 1 \\ 33 & 35 & 1 \\ 34 & 36 & 1 \\ 35 & 36 & 1 \\ 36 & 53 & 1 \\ 40 & 42 & 1 \\ 40 & 43 & 1 \\ 41 & 42 & 1 \\ 41 & 43 & 1 \\ 42 & 44 & 1 \\ 43 & 44 & 1 \\ 44 & 45 & 1 \\ 44 & 46 & 1 \\ 44 & 47 & 1 \\ 46 & 48 & 1 \\ 47 & 48 & 1 \\ 48 & 49 & 1 \\ 48 & 50 & 1 \\ 49 & 51 & 1 \\ 49 & 52 & 1 \\ 50 & 51 & 1 \\ 50 & 52 & 1 \\ 51 & 53 & 1 \\ 52 & 53 & 1 \\ 55 & 57 & 1 \\ 55 & 58 & 1 \\ 56 & 57 & 1 \\ 56 & 58 & 1 \\ 57 & 59 & 1 \\ 58 & 59 & 1 \\ 60 & 61 & 1 \\ & & \\ \text { angles }] & \\ ; \text { ai aj ak funct } \\ 2 & 1 & 29 & 5 \\ 2 & 1 & 30 & 5 \\ 2 & 1 & 31 & 5 \\ 29 & 1 & 30 & 5 \\ 29 & 1 & 31 & 5 \\ 30 & 1 & 31 & 5 \\ 1 & 2 & 17 & 5 \\ 1 & 2 & 3 & 5 \\ 1 & 2 & 21 & 5 \\ & & \end{array}$




$\begin{array}{cccc}17 & 2 & 3 & 5 \\ 17 & 2 & 21 & 5 \\ 3 & 2 & 21 & 5 \\ 33 & 3 & 2 & 5 \\ 33 & 3 & 4 & 5 \\ 33 & 3 & 32 & 5 \\ 2 & 3 & 4 & 5 \\ 2 & 3 & 32 & 5 \\ 4 & 3 & 32 & 5 \\ 34 & 4 & 3 & 5 \\ 34 & 4 & 5 & 5 \\ 34 & 4 & 35 & 5 \\ 3 & 4 & 5 & 5 \\ 3 & 4 & 35 & 5 \\ 5 & 4 & 35 & 5 \\ 36 & 5 & 4 & 5 \\ 36 & 5 & 6 & 5 \\ 36 & 5 & 16 & 5 \\ 4 & 5 & 6 & 5 \\ 4 & 5 & 16 & 5 \\ 6 & 5 & 16 & 5 \\ 13 & 6 & 5 & 5 \\ 13 & 6 & 7 & 5 \\ 13 & 6 & 8 & 5 \\ 5 & 6 & 7 & 5 \\ 5 & 6 & 8 & 5 \\ 7 & 6 & 8 & 5 \\ 37 & 7 & 6 & 5 \\ 37 & 7 & 39 & 5 \\ 37 & 7 & 38 & 5 \\ 6 & 7 & 39 & 5 \\ 6 & 7 & 38 & 5 \\ 39 & 7 & 38 & 5 \\ 9 & 8 & 41 & 5 \\ 9 & 8 & 6 & 5 \\ 9 & 8 & 40 & 5 \\ 41 & 8 & 6 & 5 \\ 41 & 8 & 40 & 5 \\ 6 & 8 & 40 & 5 \\ 10 & 9 & 43 & 5 \\ 10 & 9 & 42 & 5 \\ 10 & 9 & 8 & 5 \\ 43 & 9 & 42 & 5 \\ 43 & 9 & 8 & 5 \\ 42 & 9 & 8 & 5 \\ 9 & 10 & 44 & 5 \\ & & & \end{array}$




$\begin{array}{cccc}9 & 10 & 11 & 5 \\ 9 & 10 & 12 & 5 \\ 44 & 10 & 11 & 5 \\ 44 & 10 & 12 & 5 \\ 11 & 10 & 12 & 5 \\ 10 & 11 & 45 & 5 \\ 10 & 12 & 13 & 5 \\ 10 & 12 & 46 & 5 \\ 10 & 12 & 47 & 5 \\ 13 & 12 & 46 & 5 \\ 13 & 12 & 47 & 5 \\ 46 & 12 & 47 & 5 \\ 48 & 13 & 12 & 5 \\ 48 & 13 & 6 & 5 \\ 48 & 13 & 14 & 5 \\ 12 & 13 & 6 & 5 \\ 12 & 13 & 14 & 5 \\ 6 & 13 & 14 & 5 \\ 49 & 14 & 50 & 5 \\ 49 & 14 & 13 & 5 \\ 49 & 14 & 15 & 5 \\ 50 & 14 & 13 & 5 \\ 50 & 14 & 15 & 5 \\ 13 & 14 & 15 & 5 \\ 51 & 15 & 52 & 5 \\ 51 & 15 & 14 & 5 \\ 51 & 15 & 16 & 5 \\ 52 & 15 & 14 & 5 \\ 52 & 15 & 16 & 5 \\ 14 & 15 & 16 & 5 \\ 17 & 16 & 53 & 5 \\ 17 & 16 & 5 & 5 \\ 17 & 16 & 15 & 5 \\ 53 & 16 & 5 & 5 \\ 53 & 16 & 15 & 5 \\ 5 & 16 & 15 & 5 \\ 2 & 17 & 19 & 5 \\ 2 & 17 & 18 & 5 \\ 2 & 17 & 16 & 5 \\ 19 & 17 & 18 & 5 \\ 19 & 17 & 16 & 5 \\ 18 & 17 & 16 & 5 \\ 17 & 18 & 54 & 5 \\ 17 & 19 & 20 & 5 \\ 17 & 19 & 55 & 5 \\ 17 & 19 & 56 & 5\end{array}$




$$
\begin{array}{cccc}
20 & 19 & 55 & 5 \\
20 & 19 & 56 & 5 \\
55 & 19 & 56 & 5 \\
57 & 20 & 58 & 5 \\
57 & 20 & 19 & 5 \\
57 & 20 & 21 & 5 \\
58 & 20 & 19 & 5 \\
58 & 20 & 21 & 5 \\
19 & 20 & 21 & 5 \\
2 & 21 & 59 & 5 \\
2 & 21 & 20 & 5 \\
2 & 21 & 22 & 5 \\
59 & 21 & 20 & 5 \\
59 & 21 & 22 & 5 \\
20 & 21 & 22 & 5 \\
28 & 22 & 21 & 5 \\
28 & 22 & 23 & 5 \\
21 & 22 & 23 & 5 \\
60 & 23 & 22 & 5 \\
60 & 23 & 24 & 5 \\
22 & 23 & 24 & 5 \\
25 & 24 & 61 & 5 \\
25 & 24 & 23 & 5 \\
61 & 24 & 23 & 5 \\
26 & 25 & 27 & 5 \\
26 & 25 & 24 & 5 \\
27 & 25 & 24 & 5 \\
25 & 27 & 28 & 5 \\
27 & 28 & 22 & 5 \\
27 & 28 & 62 & 5 \\
22 & 28 & 62 & 5
\end{array}
$$

[ dihedrals ]

; ai aj ak al funct $\mathrm{c} 0 \mathrm{c} 1 \mathrm{c} 2 \mathrm{c} 3 \mathrm{c} 4 \mathrm{c} 5$

$\begin{array}{ccccc}29 & 1 & 2 & 17 & 9 \\ 29 & 1 & 2 & 3 & 9 \\ 29 & 1 & 2 & 21 & 9 \\ 30 & 1 & 2 & 17 & 9 \\ 30 & 1 & 2 & 3 & 9 \\ 30 & 1 & 2 & 21 & 9 \\ 31 & 1 & 2 & 17 & 9 \\ 31 & 1 & 2 & 3 & 9 \\ 31 & 1 & 2 & 21 & 9 \\ 1 & 2 & 17 & 19 & 9 \\ 1 & 2 & 17 & 18 & 9 \\ 1 & 2 & 17 & 16 & 9\end{array}$




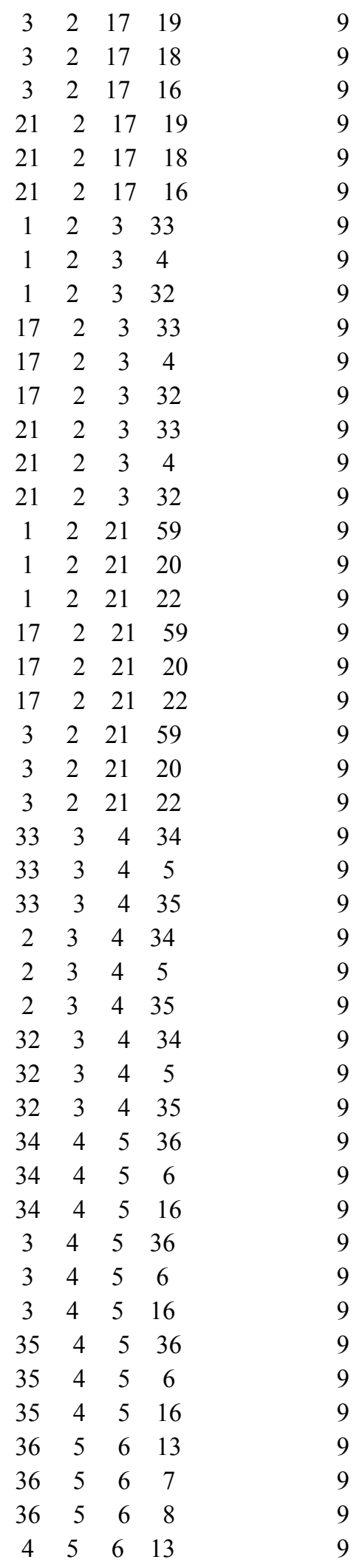




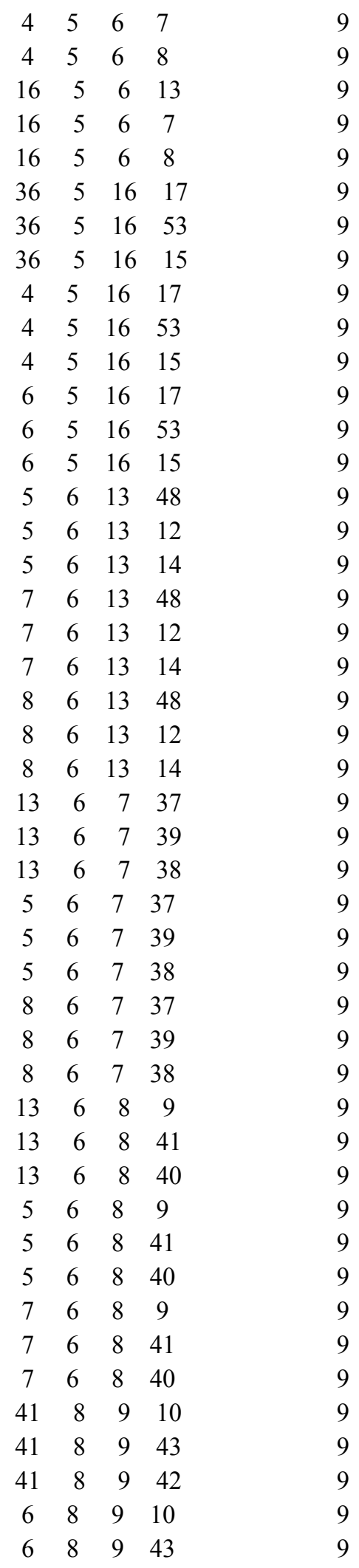




\begin{tabular}{|c|c|c|c|c|}
\hline 6 & 8 & 9 & 42 & 9 \\
\hline 40 & 8 & 9 & 10 & 9 \\
\hline 40 & 8 & 9 & 43 & 9 \\
\hline 40 & 8 & 9 & 42 & 9 \\
\hline 43 & 9 & 10 & 44 & \\
\hline 43 & 9 & 10 & 11 & \\
\hline 43 & 9 & 10 & 12 & 9 \\
\hline 42 & 9 & 10 & 44 & \\
\hline 42 & 9 & 10 & 11 & \\
\hline 42 & 9 & 10 & 12 & 9 \\
\hline 8 & 9 & 10 & 44 & 9 \\
\hline 8 & 9 & 10 & 11 & 9 \\
\hline 8 & 9 & 10 & 12 & 9 \\
\hline 9 & 10 & 11 & 45 & 9 \\
\hline 44 & 10 & 11 & 45 & 9 \\
\hline 12 & 10 & 11 & 45 & 9 \\
\hline 9 & 10 & 12 & 13 & 9 \\
\hline 9 & 10 & 12 & 46 & 9 \\
\hline 9 & 10 & 12 & 47 & 9 \\
\hline 44 & 10 & 12 & 13 & 9 \\
\hline 44 & 10 & 12 & 46 & 9 \\
\hline 44 & 10 & 12 & 47 & 9 \\
\hline 11 & 10 & 12 & 13 & 9 \\
\hline 11 & 10 & 12 & 46 & 9 \\
\hline 11 & 10 & 12 & 47 & 9 \\
\hline 10 & 12 & 13 & 48 & 9 \\
\hline 10 & 12 & 13 & 6 & 9 \\
\hline 10 & 12 & 13 & 14 & 9 \\
\hline 46 & 12 & 13 & 48 & 9 \\
\hline 46 & 12 & 13 & 6 & 9 \\
\hline 46 & 12 & 13 & 14 & 9 \\
\hline 47 & 12 & 13 & 48 & 9 \\
\hline 47 & 12 & 13 & 6 & 9 \\
\hline 47 & 12 & 13 & 14 & 9 \\
\hline 48 & 13 & 14 & 49 & 9 \\
\hline 48 & 13 & 14 & 50 & 9 \\
\hline 48 & 13 & 14 & 15 & 9 \\
\hline 12 & 13 & 14 & 49 & 9 \\
\hline 12 & 13 & 14 & 50 & 9 \\
\hline 12 & 13 & 14 & 15 & \\
\hline 6 & 13 & 14 & 49 & 9 \\
\hline 6 & 13 & 14 & 50 & 9 \\
\hline 6 & 13 & 14 & 15 & 9 \\
\hline 49 & 14 & 15 & 51 & \\
\hline 49 & 14 & 15 & 52 & 9 \\
\hline 49 & 14 & 15 & 16 & 9 \\
\hline
\end{tabular}




\begin{tabular}{|c|c|c|c|c|}
\hline 50 & 14 & 15 & 51 & 9 \\
\hline 50 & 14 & 15 & 52 & 9 \\
\hline 50 & 14 & 15 & 16 & 9 \\
\hline 13 & 14 & 15 & 51 & 9 \\
\hline 13 & 14 & 15 & 52 & 9 \\
\hline 13 & 14 & 15 & 16 & \\
\hline 51 & 15 & 16 & 17 & 9 \\
\hline 51 & 15 & 16 & 53 & 9 \\
\hline 51 & 15 & 16 & 5 & \\
\hline 52 & 15 & 16 & 17 & 9 \\
\hline 52 & 15 & 16 & 53 & 9 \\
\hline 52 & 15 & 16 & 5 & 9 \\
\hline 14 & 15 & 16 & 17 & 9 \\
\hline 14 & 15 & 16 & 53 & 9 \\
\hline 14 & 15 & 16 & 5 & 9 \\
\hline 53 & 16 & 17 & 2 & 9 \\
\hline 53 & 16 & 17 & 19 & 9 \\
\hline 53 & 16 & 17 & 18 & 9 \\
\hline 5 & 16 & 17 & 2 & 9 \\
\hline 5 & 16 & 17 & 19 & 9 \\
\hline 5 & 16 & 17 & 18 & 9 \\
\hline 15 & 16 & 17 & 2 & 9 \\
\hline 15 & 16 & 17 & 19 & 9 \\
\hline 15 & 16 & 17 & 18 & 9 \\
\hline 2 & 17 & 19 & 20 & 9 \\
\hline 2 & 17 & 19 & 55 & 9 \\
\hline 2 & 17 & 19 & 56 & 9 \\
\hline 18 & 17 & 19 & 20 & 9 \\
\hline 18 & 17 & 19 & 55 & 9 \\
\hline 18 & 17 & 19 & 56 & 9 \\
\hline 16 & 17 & 19 & 20 & 9 \\
\hline 16 & 17 & 19 & 55 & 9 \\
\hline 16 & 17 & 19 & 56 & 9 \\
\hline 2 & 17 & 18 & 54 & 9 \\
\hline 19 & 17 & 18 & 54 & 9 \\
\hline 16 & 17 & 18 & 54 & 9 \\
\hline 17 & 19 & 20 & 57 & 9 \\
\hline 17 & 19 & 20 & 58 & 9 \\
\hline 17 & 19 & 20 & 21 & 9 \\
\hline 55 & 19 & 20 & 57 & 9 \\
\hline 55 & 19 & 20 & 58 & 9 \\
\hline 55 & 19 & 20 & 21 & 9 \\
\hline 56 & 19 & 20 & 57 & 9 \\
\hline 56 & 19 & 20 & 58 & 9 \\
\hline 56 & 19 & 20 & 21 & 9 \\
\hline 57 & 20 & 21 & 2 & \\
\hline
\end{tabular}




$\begin{array}{ccccc}57 & 20 & 21 & 59 & 9 \\ 57 & 20 & 21 & 22 & 9 \\ 58 & 20 & 21 & 2 & 9 \\ 58 & 20 & 21 & 59 & 9 \\ 58 & 20 & 21 & 22 & 9 \\ 19 & 20 & 21 & 2 & 9 \\ 19 & 20 & 21 & 59 & 9 \\ 19 & 20 & 21 & 22 & 9 \\ 2 & 21 & 22 & 28 & 9 \\ 2 & 21 & 22 & 23 & 9 \\ 59 & 21 & 22 & 28 & 9 \\ 59 & 21 & 22 & 23 & 9 \\ 20 & 21 & 22 & 28 & 9 \\ 20 & 21 & 22 & 23 & 9 \\ 21 & 22 & 28 & 27 & 9 \\ 21 & 22 & 28 & 62 & 9 \\ 23 & 22 & 28 & 27 & 9 \\ 23 & 22 & 28 & 62 & 9 \\ 28 & 22 & 23 & 60 & 9 \\ 28 & 22 & 23 & 24 & 9 \\ 21 & 22 & 23 & 60 & 9 \\ 21 & 22 & 23 & 24 & 9 \\ 60 & 23 & 24 & 25 & 9 \\ 60 & 23 & 24 & 61 & 9 \\ 22 & 23 & 24 & 25 & 9 \\ 22 & 23 & 24 & 61 & 9 \\ 61 & 24 & 25 & 26 & 9 \\ 61 & 24 & 25 & 27 & 9 \\ 23 & 24 & 25 & 26 & 9 \\ 23 & 24 & 25 & 27 & 9 \\ 26 & 25 & 27 & 28 & 9 \\ 24 & 25 & 27 & 28 & 9 \\ 25 & 27 & 28 & 22 & \\ 25 & 27 & 28 & 62 & 9 \\ & & & & 9 \\ 5 & \\ 5 & \\ 5 & \end{array}$

[ dihedrals ]

; ai aj ak al funct $\mathrm{c} 0 \mathrm{c} 1 \mathrm{c} 2 \mathrm{c} 3$ $\begin{array}{lllll}25 & 24 & 26 & 27 & 2\end{array}$

; Include Position restraint file \#ifdef POSRES

\#include "bufalin_posres.itp" \#endif 
Supporting data file S2. Bufalin prm file.

[ bondtypes ]

\begin{tabular}{ccccc}
$; \quad \mathrm{i} \quad \mathrm{j}$ func & $\mathrm{b} 0$ & \multicolumn{2}{c}{$\mathrm{kb} ;$ penalty } \\
CG2R62 & CG3C51 & 1 & 0.14900000 & $192464.00 ; 70$ \\
CG3RC1 & OG311 & 1 & 0.14200000 & $358150.40 ; 16$
\end{tabular}

[ angletypes ]

; $\mathrm{i} j \mathrm{j}$ func theta0 ktheta ub0 kub; penalty

$\begin{array}{llllllll}\text { CG2R62 } & \text { CG2R62 } & \text { CG3C51 } & 5 & 124.200000 & 334.720000 & 0.00000000 & 0.00 ; 10.9\end{array}$

$\begin{array}{lllllllll}\text { OG3R60 } & \text { CG2R62 } & \text { HGR62 } & 5 & 119.000000 & 292.880000 & 0.00000000 & 0.00 ; 99.5\end{array}$

$\begin{array}{llllllll}\text { CG2R62 } & \text { CG3C51 } & \text { CG3C52 } & 5 & 112.300000 & 435.136000 & 0.00000000 & 0.00 ; 30\end{array}$

$\begin{array}{llllllll}\text { CG2R62 } & \text { CG3C51 } & \text { CG3RC1 } & 5 & 112.300000 & 435.136000 & 0.00000000 & 0.00 ; 31.1\end{array}$

$\begin{array}{llllllll}\text { CG2R62 CG3C51 HGA1 } 5 & 112.000000 & 418.400000 & 0.00000000 & 0.00 ; 30\end{array}$

$\begin{array}{lllllllll}\text { CG311 CG3RC1 OG311 } & 5 & 113.500000 & 488.272800 & 0.25610000 & 9338.69 ; 45.9\end{array}$

$\begin{array}{lllllllll}\text { CG3C52 } & \text { CG3RC1 } & \text { OG311 } & 5 & 110.000000 & 633.457600 & 0.00000000 & 0.00 ; 16\end{array}$

$\begin{array}{lllllllll}\text { CG3RC1 } & \text { CG3RC1 } & \text { OG311 } & 5 & 111.000000 & 446.432800 & 0.25610000 & 6694.40 ; 45\end{array}$

$\begin{array}{lllllllll}\text { CG3RC1 } & \text { OG311 } & \text { HGP1 } & 5 & 109.000000 & 418.400000 & 0.00000000 & 0.00 ; 1.5\end{array}$

[ dihedraltypes ]

\begin{tabular}{|c|c|c|c|c|c|c|}
\hline $\mathrm{i}$ & $\mathrm{k}$ & 1 func & phi0 & kphi & & \\
\hline CG2R62 & CG2R62 & CG2R62 & CG3C51 & 180.000000 & 12.97040 & $2 ; 20.5$ \\
\hline CG3C51 & CG2R62 & CG2R62 & OG3R60 & 180.000000 & 10.04160 & $2 ; 27.4$ \\
\hline CG3C51 & CG2R62 & CG2R62 & HGR62 & $\begin{array}{ll}9 & 180.000000\end{array}$ & 16.736000 & $2 ; 10.9$ \\
\hline CG2R62 & CG2R62 & CG3C51 & CG3C52 & 180.000000 & 0.962320 & $2 ; 100.5$ \\
\hline CG2R62 & CG2R62 & CG3C51 & CG3RC1 & $9 \quad 180.000000$ & 0.962320 & $2 ; 104$ \\
\hline CG2R62 & CG2R62 & CG3C51 & HGA1 & $9 \quad 0.000000$ & 0.008368 & $6 ; 70.5$ \\
\hline HGR62 & CG2R62 & OG3R60 & CG2R63 & 0.000000 & 3.179840 & $2 ; 144$ \\
\hline CG311 & CG311 & CG3RC1 & OG311 & 0.000000 & 0.209200 & $3 ; 46.5$ \\
\hline CG321 & CG311 & CG3RC1 & OG311 & 0.000000 & 0.209200 & $3 ; 45.9$ \\
\hline HGA1 & CG311 & CG3RC1 & OG311 & 0.000000 & 0.209200 & $3 ; 45.9$ \\
\hline CG2R62 & CG3C51 & CG3C52 & CG3C52 & $\begin{array}{ll}9 & 0.000000\end{array}$ & 0.585760 & $3 ; 30$ \\
\hline CG2R62 & CG3C51 & CG3C52 & HGA2 & 0.000000 & 0.585760 & $3 ; 30$ \\
\hline CG2R62 & CG3C51 & CG3RC1 & CG321 & $9 \quad 180.000000$ & 2.092000 & $2 ; 66$ \\
\hline CG2R62 & CG3C51 & CG3RC1 & CG331 & $9 \quad 180.000000$ & 2.092000 & $2 ; 66.9$ \\
\hline CG2R62 & CG3C51 & CG3RC1 & CG3RC1 & $\begin{array}{ll}9 & 0.000000\end{array}$ & 0.627600 & $3 ; 48$ \\
\hline CG3C52 & CG3C52 & CG3RC1 & OG311 & $9 \quad 180.000000$ & 2.092000 & $1 ; 16$ \\
\hline CG3C52 & CG3C52 & CG3RC1 & OG311 & 0.000000 & 2.928800 & $2 ; 16$ \\
\hline CG3C52 & CG3C52 & CG3RC1 & OG311 & 0.000000 & 1.673600 & $3 ; 16$ \\
\hline CG3C52 & CG3C52 & CG3RC1 & OG311 & 0.000000 & 1.673600 & $5 ; 16$ \\
\hline HGA2 & CG3C52 & CG3RC1 & OG311 & $9 \quad 180.000000$ & 0.815880 & $3 ; 16$ \\
\hline CG321 & CG3RC1 & CG3RC1 & OG311 & 0.000000 & 0.627600 & $3 ; 45$ \\
\hline CG331 & CG3RC1 & CG3RC1 & OG311 & 0.000000 & 0.209200 & $3 ; 45$ \\
\hline CG3C51 & CG3RC1 & CG3RC1 & OG311 & 0.000000 & 0.209200 & $3 ; 45$ \\
\hline CG311 & CG3RC1 & OG311 & HGP1 & $0.000000 \quad 1$ & 1.213360 & $1 ; 47$ \\
\hline CG311 & CG3RC1 & OG311 & HGP1 & 0.000000 & 2.594080 & $2 ; 47$ \\
\hline CG311 & CG3RC1 & OG311 & HGP1 & 0.000000 & 0.209200 & $3 ; 47$ \\
\hline
\end{tabular}




$\begin{array}{llllllll}\text { CG3C52 } & \text { CG3RC1 } & \text { OG311 } & \text { HGP1 } & 9 & 0.000000 & 1.213360 & 1 ; 16 \\ \text { CG3C52 } & \text { CG3RC1 } & \text { OG311 } & \text { HGP1 } & 9 & 0.000000 & 2.594080 & 2 ; 16 \\ \text { CG3C52 } & \text { CG3RC1 } & \text { OG311 } & \text { HGP1 } & 9 & 0.000000 & 0.209200 & 3 ; 16 \\ \text { CG3RC1 } & \text { CG3RC1 } & \text { OG311 } & \text { HGP1 } & 9 & 0.000000 & 6.276000 & 1 ; 56 \\ \text { CG3RC1 } & \text { CG3RC1 } & \text { OG311 } & \text { HGP1 } & 9 & 180.000000 & 1.255200 & 2 ; 56 \\ \text { CG3RC1 } & \text { CG3RC1 } & \text { OG311 } & \text { HGP1 } & 9 & 0.000000 & 1.338880 & 3 ; 56\end{array}$

[ dihedraltypes ]

; 'improper' dihedrals

; $\mathrm{i}$ j $\mathrm{k} \quad \mathrm{l}$ func phi0 kphi 
Supporting data file S3. Cinobufagin itp file.

[ moleculetype ]

; Name nrexcl

cinobufagin 3

[ atoms ]

; $\mathrm{nr}$ type resnr residue atom cgnr charge mass typeB chargeB massB

; residue 1 cinobufagin rtp cinobufagin q qsum; charge_penalty

\begin{tabular}{|c|c|c|c|c|c|c|c|}
\hline & CG331 & 1 cinobufagin & $\mathrm{C} 18$ & 1 & -0.269 & 12.011 ; & ; 4.089 \\
\hline & CG3RC1 & 1 cinobufagin & C13 & 2 & 0.003 & 12.011 & ; 27.193 \\
\hline & CG321 & 1 cinobufagin & $\mathrm{C} 12$ & 3 & -0.183 & 12.011 ; & ; 4.089 \\
\hline & CG321 & 1 cinobufagin & $\mathrm{C} 11$ & 4 & -0.182 & $12.011 ;$ & \\
\hline & CG311 & 1 cinobufagin & C9 & 5 & -0.091 & $12.011 ; 2$ & 2.500 \\
\hline & CG301 & 1 cinobufagin & $\mathrm{C} 10$ & 6 & -0.001 & 12.011 ; & ; \\
\hline & CG331 & 1 cinobufagin & $\mathrm{C} 19$ & 7 & -0.274 & 12.011 & ; \\
\hline & CG321 & 1 cinobufagin & $\mathrm{C} 1$ & 8 & -0.180 & 12.011 & \\
\hline & CG321 & 1 cinobufagin & $\mathrm{C} 2$ & 9 & -0.179 & $12.011 ;$ & \\
\hline & CG311 & 1 cinobufagin & $\mathrm{C} 3$ & 10 & 0.135 & 12.011 ; & ; \\
\hline & OG311 & 1 cinobufagin & $\mathrm{O} 3$ & 11 & -0.650 & 15.999 & ; \\
\hline & CG321 & 1 cinobufagin & $\mathrm{C} 4$ & 12 & -0.177 & 12.011 & ; \\
\hline & CG311 & 1 cinobufagin & $\mathrm{C} 5$ & 13 & -0.090 & 12.011 & ; \\
\hline & CG321 & 1 cinobufagin & $\mathrm{C} 6$ & 14 & -0.181 & 12.011 & ; \\
\hline & CG321 & 1 cinobufagin & $\mathrm{C} 7$ & 15 & -0.182 & $12.011 ;$ & ; 2.500 \\
\hline 1 & CG311 & 1 cinobufagin & $\mathrm{C} 8$ & 16 & -0.086 & $12.011 ;$ & ;17.655 \\
\hline 17 & CG3C51 & 1 cinobufagin & $\mathrm{C} 16$ & 17 & 0.051 & 12.011 & ;25.744 \\
\hline 18 & CG3C51 & 1 cinobufagin & $\mathrm{C} 17$ & 18 & -0.033 & 12.011 & ;72.189 \\
\hline 19 & CG2R62 & 1 cinobufagin & $\mathrm{C} 20$ & 19 & 0.031 & 12.011 & ;71.726 \\
\hline 20 & CG2R62 & 1 cinobufagin & $\mathrm{C} 22$ & 20 & -0.272 & 12.011 & $; 12.532$ \\
\hline 2 & CG2R62 & 1 cinobufagin & $\mathrm{C} 23$ & 21 & -0.347 & 12.011 & ;8.966 \\
\hline 22 & CG2R63 & 1 cinobufagin & $\mathrm{C} 24$ & 22 & 0.490 & 12.011 & $; 2.500$ \\
\hline 23 & OG2D4 & 1 cinobufagin & $\mathrm{O} 24$ & 23 & -0.460 & 15.999 & ; \\
\hline 24 & OG3R60 & 1 cinobufagin & $\mathrm{O} 21$ & 24 & -0.362 & 15.999 & 9;59.051 \\
\hline 25 & CG2R62 & 1 cinobufagin & $\mathrm{C} 21$ & 25 & 0.119 & 12.011 & ;84.241 \\
\hline 26 & HGA3 & 1 cinobufagin & H181 & 26 & 0.090 & 1.008 & ; \\
\hline 27 & HGA3 & 1 cinobufagin & H182 & 27 & 0.090 & 1.008 & ; \\
\hline 28 & HGA3 & 1 cinobufagin & H183 & 28 & 0.090 & 1.008 & ; \\
\hline 29 & HGA2 & 1 cinobufagin & H121 & 29 & 0.090 & 1.008 & ; \\
\hline 30 & HGA2 & 1 cinobufagin & H122 & 30 & 0.090 & 1.008 & \\
\hline 31 & HGA2 & 1 cinobufagin & H111 & 31 & 0.090 & 1.008 & ; \\
\hline 32 & HGA2 & 1 cinobufagin & H112 & 32 & 0.090 & 1.008 & ; \\
\hline 33 & HGA1 & 1 cinobufagin & H9 & 33 & 0.090 & $1.008 ;$ & \\
\hline 34 & HGA3 & 1 cinobufagin & H191 & 34 & 0.090 & 1.008 & \\
\hline 35 & HGA3 & 1 cinobufagin & H192 & 35 & 0.090 & 1.008 & ; \\
\hline 36 & HGA3 & 1 cinobufagin & H193 & 36 & 0.090 & 1.008 & ; \\
\hline 37 & HGA2 & 1 cinobufagin & H11 & 37 & 0.090 & 1.008 & ; \\
\hline & HGA2 & 1 cinobufagin & H12 & 38 & 0.090 & 1.008 & ; \\
\hline
\end{tabular}




$\begin{array}{llllllll}39 & \text { HGA2 } & 1 \text { cinobufagin } & \text { H21 } & 39 & 0.090 & 1.008 ; \\ 40 & \text { HGA2 } & 1 \text { cinobufagin } & \text { H22 } & 40 & 0.090 & 1.008 & ; \\ 41 & \text { HGA1 } & 1 \text { cinobufagin } & \text { H3 } & 41 & 0.090 & 1.008 & ; \\ 42 & \text { HGP1 } & 1 \text { cinobufagin } & \text { HO3 } & 42 & 0.419 & 1.008 & ; \\ 43 & \text { HGA2 } & 1 \text { cinobufagin } & \text { H41 } & 43 & 0.090 & 1.008 & ; \\ 44 & \text { HGA2 } & 1 \text { cinobufagin } & \text { H42 } & 44 & 0.090 & 1.008 & ; \\ 45 & \text { HGA1 } & 1 \text { cinobufagin } & \text { H5 } & 45 & 0.090 & 1.008 ; \\ 46 & \text { HGA2 } & 1 \text { cinobufagin } & \text { H61 } & 46 & 0.090 & 1.008 & ; \\ 47 & \text { HGA2 } & 1 \text { cinobufagin } & \text { H62 } & 47 & 0.090 & 1.008 & ; \\ 48 & \text { HGA2 } & 1 \text { cinobufagin } & \text { H71 } & 48 & 0.090 & 1.008 & ; \\ 49 & \text { HGA2 } & 1 \text { cinobufagin } & \text { H72 } & 49 & 0.090 & 1.008 & ; \\ 50 & \text { HGA1 } & 1 \text { cinobufagin } & \text { H8 } & 50 & 0.090 & 1.008 & ; 2.175 \\ 51 & \text { HGA1 } & 1 \text { cinobufagin } & \text { H161 } & 51 & 0.090 & 1.008 & ; 3.456 \\ 52 & \text { OG302 } & 1 \text { cinobufagin } & \text { O52 } & 52 & -0.370 & 15.999 & ; 8.514 \\ 53 & \text { HGA1 } & 1 \text { cinobufagin } & \text { H17 } & 53 & 0.090 & 1.008 & ; 3.841 \\ 54 & \text { HGR62 } & 1 \text { cinobufagin } & \text { H2 } & 54 & 0.268 & 1.008 & ; 6.142 \\ 55 & \text { HGR62 } & 1 \text { cinobufagin } & \text { H23 } & 55 & 0.199 & 1.008 & ; \\ 56 & \text { HGR62 } & 1 \text { cinobufagin } & \text { H1 } & 56 & 0.284 & 1.008 ; 84.726 \\ 57 & \text { CG3RC1 } & 1 \text { cinobufagin } & \text { C57 } & 57 & 0.191 & 12.011 & ; 95.862 \\ 58 & \text { CG3RC1 } & 1 \text { cinobufagin } & \text { C58 } & 58 & 0.124 & 12.011 & ; 95.023 \\ 59 & \text { OG3C31 } & 1 \text { cinobufagin } & \text { O59 } & 59 & -0.404 & 15.999 & ; 133.011 \\ 60 & \text { HGA1 } & 1 \text { cinobufagin } & \text { H60 } & 60 & 0.090 & 1.008 & ; 4.678 \\ 61 & \text { CG2O2 } & 1 \text { cinobufagin } & \text { C61 } & 61 & 0.897 & 12.011 ; 8.127 \\ 62 & \text { CG331 } & 1 \text { cinobufagin } & \text { C62 } & 62 & -0.311 & 12.011 & ; 3.162 \\ 63 & \text { OG2D1 } & 1 \text { cinobufagin } & \text { O63 } & 63 & -0.627 & 15.999 & ; 4.374 \\ 64 & \text { HGA3 } & 1 \text { cinobufagin } & \text { H64 } & 64 & 0.090 & 1.008 & ; \\ 65 & \text { HGA3 } & 1 \text { cinobufagin } & \text { H65 } & 65 & 0.090 & 1.008 & ; \\ 66 & \text { HGA3 } & 1 \text { cinobufagin } & \text { H66 } & 66 & 0.090 & 1.008 & ;\end{array}$

[bonds ]

; ai aj funct $\mathrm{c} 0 \mathrm{c} 1 \mathrm{c} 2 \mathrm{c} 3$

$\begin{array}{ccc}1 & 2 & 1 \\ 1 & 27 & 1 \\ 1 & 28 & 1 \\ 1 & 26 & 1 \\ 2 & 18 & 1 \\ 2 & 3 & 1 \\ 2 & 57 & 1 \\ 3 & 4 & 1 \\ 3 & 29 & 1 \\ 3 & 30 & 1 \\ 4 & 5 & 1 \\ 4 & 31 & 1 \\ 4 & 32 & 1 \\ 5 & 33 & 1 \\ 5 & 6 & 1\end{array}$




$\begin{array}{ccc}5 & 16 & 1 \\ 6 & 13 & 1 \\ 6 & 7 & 1 \\ 6 & 8 & 1 \\ 7 & 34 & 1 \\ 7 & 35 & 1 \\ 7 & 36 & 1 \\ 8 & 9 & 1 \\ 8 & 37 & 1 \\ 8 & 38 & 1 \\ 9 & 10 & 1 \\ 9 & 40 & 1 \\ 9 & 39 & 1 \\ 10 & 41 & 1 \\ 10 & 11 & 1 \\ 10 & 12 & 1 \\ 11 & 42 & 1 \\ 12 & 43 & 1 \\ 12 & 44 & 1 \\ 12 & 13 & 1 \\ 13 & 45 & 1 \\ 13 & 14 & 1 \\ 14 & 47 & 1 \\ 14 & 46 & 1 \\ 14 & 15 & 1 \\ 15 & 49 & 1 \\ 15 & 48 & 1 \\ 15 & 16 & 1 \\ 16 & 57 & 1 \\ 16 & 50 & 1 \\ 17 & 18 & 1 \\ 17 & 51 & 1 \\ 17 & 52 & 1 \\ 17 & 58 & 1 \\ 18 & 19 & 1 \\ 18 & 53 & 1 \\ 19 & 25 & 1 \\ 19 & 20 & 1 \\ 20 & 21 & 1 \\ 20 & 54 & 1 \\ 21 & 22 & 1 \\ 21 & 55 & 1 \\ 22 & 23 & 1 \\ 22 & 24 & 1 \\ 24 & 25 & 1 \\ 25 & 56 & 1 \\ & & \end{array}$




$\begin{array}{lll}52 & 61 & 1 \\ 57 & 59 & 1 \\ 57 & 58 & 1 \\ 58 & 59 & 1 \\ 58 & 60 & 1 \\ 61 & 62 & 1 \\ 61 & 63 & 1 \\ 62 & 65 & 1 \\ 62 & 66 & 1 \\ 62 & 64 & 1\end{array}$

[pairs ]

; ai aj funct $\mathrm{c} 0 \mathrm{c} 1 \mathrm{c} 2 \mathrm{c} 3$

$\begin{array}{lll}1 & 4 & 1\end{array}$

$\begin{array}{lll}1 & 16 & 1\end{array}$

$\begin{array}{lll}1 & 17 & 1\end{array}$

$\begin{array}{lll}1 & 19 & 1\end{array}$

$\begin{array}{lll}1 & 53 & 1\end{array}$

$\begin{array}{lll}1 & 58 & 1\end{array}$

$\begin{array}{lll}1 & 59 & 1\end{array}$

$\begin{array}{lll}1 & 29 & 1\end{array}$

$\begin{array}{lll}1 & 30 & 1\end{array}$

$\begin{array}{lll}2 & 20 & 1\end{array}$

$\begin{array}{lll}2 & 5 & 1\end{array}$

$\begin{array}{lll}2 & 15 & 1\end{array}$

$\begin{array}{lll}2 & 50 & 1\end{array}$

$\begin{array}{lll}2 & 51 & 1\end{array}$

$\begin{array}{lll}2 & 52 & 1\end{array}$

$\begin{array}{lll}2 & 25 & 1\end{array}$

$\begin{array}{lll}2 & 60 & 1\end{array}$

$\begin{array}{lll}2 & 31 & 1\end{array}$

$\begin{array}{lll}2 & 32 & 1\end{array}$

$\begin{array}{lll}3 & 33 & 1\end{array}$

$\begin{array}{lll}3 & 6 & 1\end{array}$

$\begin{array}{lll}3 & 16 & 1\end{array}$

$\begin{array}{lll}3 & 17 & 1\end{array}$

$\begin{array}{lll}3 & 19 & 1\end{array}$

$\begin{array}{lll}3 & 53 & 1\end{array}$

$\begin{array}{lll}3 & 58 & 1\end{array}$

$\begin{array}{lll}3 & 26 & 1\end{array}$

$\begin{array}{lll}3 & 27 & 1\end{array}$

$\begin{array}{lll}3 & 28 & 1\end{array}$

$\begin{array}{lll}3 & 59 & 1\end{array}$

$\begin{array}{lll}4 & 50 & 1\end{array}$

$\begin{array}{lll}4 & 7 & 1\end{array}$

$\begin{array}{lll}4 & 8 & 1\end{array}$ 


$\begin{array}{ccc}4 & 13 & 1 \\ 4 & 15 & 1 \\ 4 & 18 & 1 \\ 4 & 57 & 1 \\ 5 & 35 & 1 \\ 5 & 36 & 1 \\ 5 & 37 & 1 \\ 5 & 38 & 1 \\ 5 & 34 & 1 \\ 5 & 9 & 1 \\ 5 & 12 & 1 \\ 5 & 45 & 1 \\ 5 & 14 & 1 \\ 5 & 48 & 1 \\ 5 & 49 & 1 \\ 5 & 58 & 1 \\ 5 & 59 & 1 \\ 5 & 29 & 1 \\ 5 & 30 & 1 \\ 6 & 39 & 1 \\ 6 & 40 & 1 \\ 6 & 10 & 1 \\ 6 & 43 & 1 \\ 6 & 44 & 1 \\ 6 & 46 & 1 \\ 6 & 15 & 1 \\ 6 & 50 & 1 \\ 6 & 47 & 1 \\ 6 & 57 & 1 \\ 6 & 31 & 1 \\ 6 & 32 & 1 \\ 7 & 33 & 1 \\ 7 & 37 & 1 \\ 7 & 38 & 1 \\ 7 & 9 & 1 \\ 7 & 12 & 1 \\ 7 & 45 & 1 \\ 7 & 14 & 1 \\ 7 & 16 & 1 \\ 8 & 33 & 1 \\ 8 & 34 & 1 \\ 8 & 35 & 1 \\ 8 & 41 & 1 \\ 8 & 11 & 1 \\ 8 & 12 & 1 \\ 8 & 45 & 1\end{array}$




$\begin{array}{ccc}8 & 14 & 1 \\ 8 & 16 & 1 \\ 8 & 36 & 1 \\ 9 & 42 & 1 \\ 9 & 43 & 1 \\ 9 & 44 & 1 \\ 9 & 13 & 1 \\ 10 & 45 & 1 \\ 10 & 14 & 1 \\ 10 & 37 & 1 \\ 10 & 38 & 1 \\ 11 & 39 & 1 \\ 11 & 43 & 1 \\ 11 & 44 & 1 \\ 11 & 13 & 1 \\ 11 & 40 & 1 \\ 12 & 39 & 1 \\ 12 & 40 & 1 \\ 12 & 42 & 1 \\ 12 & 46 & 1 \\ 12 & 47 & 1 \\ 12 & 15 & 1 \\ 13 & 33 & 1 \\ 13 & 34 & 1 \\ 13 & 35 & 1 \\ 13 & 37 & 1 \\ 13 & 38 & 1 \\ 13 & 16 & 1 \\ 13 & 49 & 1 \\ 13 & 41 & 1 \\ 13 & 36 & 1 \\ 13 & 48 & 1 \\ 14 & 43 & 1 \\ 14 & 44 & 1 \\ 14 & 50 & 1 \\ 14 & 57 & 1 \\ 15 & 33 & 1 \\ 15 & 45 & 1 \\ 15 & 58 & 1 \\ 15 & 59 & 1 \\ 16 & 46 & 1 \\ 16 & 47 & 1 \\ 16 & 17 & 1 \\ 16 & 18 & 1 \\ 16 & 60 & 1 \\ 16 & 31 & 1 \\ & & \end{array}$




$\begin{array}{lll}16 & 32 & 1 \\ 17 & 20 & 1 \\ 17 & 25 & 1 \\ 17 & 62 & 1 \\ 17 & 63 & 1 \\ 18 & 61 & 1 \\ 18 & 24 & 1 \\ 18 & 60 & 1 \\ 18 & 59 & 1 \\ 18 & 21 & 1 \\ 18 & 54 & 1 \\ 18 & 56 & 1 \\ 18 & 26 & 1 \\ 18 & 27 & 1 \\ 18 & 28 & 1 \\ 18 & 29 & 1 \\ 18 & 30 & 1 \\ 19 & 51 & 1 \\ 19 & 52 & 1 \\ 19 & 22 & 1 \\ 19 & 55 & 1 \\ 19 & 57 & 1 \\ 19 & 58 & 1 \\ 20 & 56 & 1 \\ 20 & 53 & 1 \\ 20 & 23 & 1 \\ 20 & 24 & 1 \\ 21 & 25 & 1 \\ 22 & 54 & 1 \\ 22 & 56 & 1 \\ 23 & 25 & 1 \\ 23 & 55 & 1 \\ 24 & 55 & 1 \\ 25 & 53 & 1 \\ 25 & 54 & 1 \\ 26 & 57 & 1 \\ 27 & 57 & 1 \\ 28 & 57 & 1 \\ 29 & 57 & 1 \\ 29 & 31 & 1 \\ 29 & 32 & 1 \\ 30 & 57 & 1 \\ 30 & 31 & 1 \\ 30 & 32 & 1 \\ 31 & 33 & 1 \\ 32 & 33 & 1\end{array}$




$\begin{array}{lll}33 & 50 & 1 \\ 33 & 57 & 1 \\ 37 & 39 & 1 \\ 37 & 40 & 1 \\ 38 & 39 & 1 \\ 38 & 40 & 1 \\ 39 & 41 & 1 \\ 40 & 41 & 1 \\ 41 & 42 & 1 \\ 41 & 43 & 1 \\ 41 & 44 & 1 \\ 43 & 45 & 1 \\ 44 & 45 & 1 \\ 45 & 46 & 1 \\ 45 & 47 & 1 \\ 46 & 49 & 1 \\ 46 & 48 & 1 \\ 47 & 49 & 1 \\ 47 & 48 & 1 \\ 48 & 50 & 1 \\ 48 & 57 & 1 \\ 49 & 50 & 1 \\ 49 & 57 & 1 \\ 50 & 58 & 1 \\ 50 & 59 & 1 \\ 51 & 53 & 1 \\ 51 & 57 & 1 \\ 51 & 59 & 1 \\ 51 & 60 & 1 \\ 51 & 61 & 1 \\ 52 & 65 & 1 \\ 52 & 66 & 1 \\ 52 & 53 & 1 \\ 52 & 57 & 1 \\ 52 & 59 & 1 \\ 52 & 60 & 1 \\ 52 & 64 & 1 \\ 53 & 57 & 1 \\ 53 & 58 & 1 \\ 54 & 55 & 1 \\ 58 & 61 & 1 \\ 63 & 65 & 1 \\ 63 & 64 & 1 \\ 63 & 66 & 1\end{array}$

[ angles ] 
; ai aj ak funct $\mathrm{c} 0 \mathrm{c} 1 \mathrm{c} 2 \mathrm{c} 3$

$\begin{array}{llll}2 & 1 & 27 & 5\end{array}$

$\begin{array}{llll}2 & 1 & 28 & 5\end{array}$

$\begin{array}{llll}2 & 1 & 26 & 5\end{array}$

$\begin{array}{llll}27 & 1 & 28 & 5\end{array}$

$\begin{array}{llll}27 & 1 & 26 & 5\end{array}$

$\begin{array}{llll}28 & 1 & 26 & 5\end{array}$

$\begin{array}{llll}1 & 2 & 18 & 5\end{array}$

$\begin{array}{llll}1 & 2 & 3 & 5\end{array}$

$\begin{array}{llll}1 & 2 & 57 & 5\end{array}$

$\begin{array}{llll}18 & 2 & 3 & 5\end{array}$

$\begin{array}{llll}18 & 2 & 57 & 5\end{array}$

$\begin{array}{llll}3 & 2 & 57 & 5\end{array}$

$\begin{array}{llll}2 & 3 & 4 & 5\end{array}$

$\begin{array}{llll}2 & 3 & 29 & 5\end{array}$

$\begin{array}{llll}2 & 3 & 30 & 5\end{array}$

$\begin{array}{llll}4 & 3 & 29 & 5\end{array}$

$\begin{array}{llll}4 & 3 & 30 & 5\end{array}$

$\begin{array}{llll}29 & 3 & 30 & 5\end{array}$

$\begin{array}{llll}3 & 4 & 5 & 5\end{array}$

$\begin{array}{llll}3 & 4 & 31 & 5\end{array}$

$\begin{array}{llll}3 & 4 & 32 & 5\end{array}$

$\begin{array}{llll}5 & 4 & 31 & 5\end{array}$

$\begin{array}{llll}5 & 4 & 32 & 5\end{array}$

$\begin{array}{llll}31 & 4 & 32 & 5\end{array}$

$\begin{array}{llll}33 & 5 & 4 & 5\end{array}$

$\begin{array}{llll}33 & 5 & 6 & 5\end{array}$

$\begin{array}{llll}33 & 5 & 16 & 5\end{array}$

$\begin{array}{llll}4 & 5 & 6 & 5\end{array}$

$\begin{array}{llll}4 & 5 & 16 & 5\end{array}$

$\begin{array}{llll}6 & 5 & 16 & 5\end{array}$

$\begin{array}{llll}13 & 6 & 5 & 5\end{array}$

$\begin{array}{llll}13 & 6 & 7 & 5\end{array}$

$\begin{array}{llll}13 & 6 & 8 & 5\end{array}$

$\begin{array}{llll}5 & 6 & 7 & 5\end{array}$

$\begin{array}{llll}5 & 6 & 8 & 5\end{array}$

$\begin{array}{llll}7 & 6 & 8 & 5\end{array}$

$\begin{array}{llll}34 & 7 & 35 & 5\end{array}$

$\begin{array}{llll}34 & 7 & 36 & 5\end{array}$

$\begin{array}{llll}34 & 7 & 6 & 5\end{array}$

$\begin{array}{llll}35 & 7 & 36 & 5\end{array}$

$\begin{array}{llll}35 & 7 & 6 & 5\end{array}$

$\begin{array}{llll}36 & 7 & 6 & 5\end{array}$

$\begin{array}{llll}9 & 8 & 37 & 5\end{array}$

$\begin{array}{llll}9 & 8 & 6 & 5\end{array}$

$\begin{array}{llll}9 & 8 & 38 & 5\end{array}$ 


$\begin{array}{cccc}37 & 8 & 6 & 5 \\ 37 & 8 & 38 & 5 \\ 6 & 8 & 38 & 5 \\ 10 & 9 & 40 & 5 \\ 10 & 9 & 39 & 5 \\ 10 & 9 & 8 & 5 \\ 40 & 9 & 39 & 5 \\ 40 & 9 & 8 & 5 \\ 39 & 9 & 8 & 5 \\ 9 & 10 & 41 & 5 \\ 9 & 10 & 11 & 5 \\ 9 & 10 & 12 & 5 \\ 41 & 10 & 11 & 5 \\ 41 & 10 & 12 & 5 \\ 11 & 10 & 12 & 5 \\ 10 & 11 & 42 & 5 \\ 10 & 12 & 43 & 5 \\ 10 & 12 & 44 & 5 \\ 10 & 12 & 13 & 5 \\ 43 & 12 & 44 & 5 \\ 43 & 12 & 13 & 5 \\ 44 & 12 & 13 & 5 \\ 12 & 13 & 45 & 5 \\ 12 & 13 & 6 & 5 \\ 12 & 13 & 14 & 5 \\ 45 & 13 & 6 & 5 \\ 45 & 13 & 14 & 5 \\ 6 & 13 & 14 & 5 \\ 47 & 14 & 13 & 5 \\ 47 & 14 & 46 & 5 \\ 47 & 14 & 15 & 5 \\ 13 & 14 & 46 & 5 \\ 13 & 14 & 15 & 5 \\ 46 & 14 & 15 & 5 \\ 49 & 15 & 48 & 5 \\ 49 & 15 & 14 & 5 \\ 49 & 15 & 16 & 5 \\ 48 & 15 & 14 & 5 \\ 48 & 15 & 16 & 5 \\ 14 & 15 & 16 & 5 \\ 57 & 16 & 50 & 5 \\ 57 & 16 & 5 & 5 \\ 57 & 16 & 15 & 5 \\ 50 & 16 & 5 & 5 \\ 50 & 16 & 15 & 5 \\ 5 & 16 & 15 & 5\end{array}$




$\begin{array}{cccc}18 & 17 & 51 & 5 \\ 18 & 17 & 52 & 5 \\ 18 & 17 & 58 & 5 \\ 51 & 17 & 52 & 5 \\ 51 & 17 & 58 & 5 \\ 52 & 17 & 58 & 5 \\ 17 & 18 & 2 & 5 \\ 17 & 18 & 19 & 5 \\ 17 & 18 & 53 & 5 \\ 2 & 18 & 19 & 5 \\ 2 & 18 & 53 & 5 \\ 19 & 18 & 53 & 5 \\ 25 & 19 & 18 & 5 \\ 25 & 19 & 20 & 5 \\ 18 & 19 & 20 & 5 \\ 19 & 20 & 21 & 5 \\ 19 & 20 & 54 & 5 \\ 21 & 20 & 54 & 5 \\ 20 & 21 & 22 & 5 \\ 20 & 21 & 55 & 5 \\ 22 & 21 & 55 & 5 \\ 21 & 22 & 23 & 5 \\ 21 & 22 & 24 & 5 \\ 23 & 22 & 24 & 5 \\ 25 & 24 & 22 & 5 \\ 19 & 25 & 56 & 5 \\ 19 & 25 & 24 & 5 \\ 56 & 25 & 24 & 5 \\ 17 & 52 & 61 & 5 \\ 2 & 57 & 59 & 5 \\ 2 & 57 & 58 & 5 \\ 2 & 57 & 16 & 5 \\ 59 & 57 & 58 & 5 \\ 59 & 57 & 16 & 5 \\ 58 & 57 & 16 & 5 \\ 17 & 58 & 57 & 5 \\ 17 & 58 & 59 & 5 \\ 17 & 58 & 60 & 5 \\ 57 & 58 & 59 & 5 \\ 57 & 58 & 60 & 5 \\ 59 & 58 & 60 & 5 \\ 57 & 59 & 58 & 5 \\ 52 & 61 & 62 & 5 \\ 52 & 61 & 63 & 5 \\ 62 & 61 & 63 & 5 \\ 65 & 62 & 66 & 5 \\ & & & \end{array}$




$$
\begin{array}{llll}
65 & 62 & 61 & 5 \\
65 & 62 & 64 & 5 \\
66 & 62 & 61 & 5 \\
66 & 62 & 64 & 5 \\
61 & 62 & 64 & 5
\end{array}
$$

\begin{tabular}{|c|c|c|c|c|}
\hline 27 & 1 & 2 & 18 & 9 \\
\hline 27 & 1 & 2 & 3 & 9 \\
\hline 27 & 1 & 2 & 57 & 9 \\
\hline 28 & 1 & 2 & 18 & 9 \\
\hline 28 & 1 & 2 & 3 & 9 \\
\hline 28 & 1 & 2 & 57 & 9 \\
\hline 26 & 1 & 2 & 18 & 9 \\
\hline 26 & 1 & 2 & 3 & 9 \\
\hline 26 & 1 & 2 & 57 & 9 \\
\hline 1 & 2 & 18 & 17 & 9 \\
\hline 1 & 2 & 18 & 19 & 9 \\
\hline 1 & 2 & 18 & 53 & 9 \\
\hline 3 & 2 & 18 & 17 & 9 \\
\hline 3 & 2 & 18 & 19 & 9 \\
\hline 3 & 2 & 18 & 53 & 9 \\
\hline 57 & 2 & 18 & 17 & 9 \\
\hline 57 & 2 & 18 & 19 & 9 \\
\hline 57 & 2 & 18 & 53 & 9 \\
\hline 1 & 2 & 3 & 4 & 9 \\
\hline 1 & 2 & 3 & 29 & 9 \\
\hline 1 & 2 & 3 & 30 & 9 \\
\hline 18 & 2 & 3 & 4 & 9 \\
\hline 18 & 2 & 3 & 29 & 9 \\
\hline 18 & 2 & 3 & 30 & 9 \\
\hline 57 & 2 & 3 & 4 & 9 \\
\hline 57 & 2 & 3 & 29 & 9 \\
\hline 57 & 2 & 3 & 30 & 9 \\
\hline 1 & 2 & 57 & 59 & 9 \\
\hline 1 & 2 & 57 & 58 & 9 \\
\hline 1 & 2 & 57 & 16 & 9 \\
\hline 18 & 2 & 57 & 59 & 9 \\
\hline 18 & 2 & 57 & 58 & 9 \\
\hline 18 & 2 & 57 & 16 & 9 \\
\hline 3 & 2 & 57 & 59 & 9 \\
\hline 3 & 2 & 57 & 58 & 9 \\
\hline 3 & 2 & 57 & 16 & 9 \\
\hline 2 & 3 & 4 & 5 & 9 \\
\hline 2 & 3 & 4 & 31 & 9 \\
\hline
\end{tabular}

[ dihedrals ]

; ai aj ak al funct $\mathrm{c} 0 \mathrm{c} 1 \mathrm{c} 2 \mathrm{c} 3 \mathrm{c} 4 \mathrm{c5}$ 


\begin{tabular}{|c|c|c|c|c|}
\hline 2 & 3 & 4 & 32 & \\
\hline 29 & 3 & 4 & 5 & 9 \\
\hline 29 & 3 & 4 & 31 & 9 \\
\hline 29 & 3 & 4 & 32 & 9 \\
\hline 30 & 3 & 4 & 5 & \\
\hline 30 & 3 & 4 & 31 & \\
\hline 30 & 3 & 4 & 32 & 9 \\
\hline 3 & 4 & 5 & 33 & 9 \\
\hline 3 & 4 & 5 & 6 & 9 \\
\hline 3 & 4 & 5 & 16 & 9 \\
\hline 31 & 4 & 5 & 33 & 9 \\
\hline 31 & 4 & 5 & 6 & 9 \\
\hline 31 & 4 & 5 & 16 & 9 \\
\hline 32 & 4 & 5 & 33 & 9 \\
\hline 32 & 4 & 5 & 6 & 9 \\
\hline 32 & 4 & 5 & 16 & 9 \\
\hline 33 & 5 & 6 & 13 & 9 \\
\hline 33 & 5 & 6 & 7 & 9 \\
\hline 33 & 5 & 6 & 8 & 9 \\
\hline 4 & 5 & 6 & 13 & 9 \\
\hline 4 & 5 & 6 & 7 & 9 \\
\hline 4 & 5 & 6 & 8 & 9 \\
\hline 16 & 5 & 6 & 13 & 9 \\
\hline 16 & 5 & 6 & 7 & 9 \\
\hline 16 & 5 & 6 & 8 & 9 \\
\hline 33 & 5 & 16 & 57 & 9 \\
\hline 33 & 5 & 16 & 50 & 9 \\
\hline 33 & 5 & 16 & 15 & 9 \\
\hline 4 & 5 & 16 & 57 & 9 \\
\hline 4 & 5 & 16 & 50 & 9 \\
\hline 4 & 5 & 16 & 15 & 9 \\
\hline 6 & 5 & 16 & 57 & 9 \\
\hline 6 & 5 & 16 & 50 & 9 \\
\hline 6 & 5 & 16 & 15 & 9 \\
\hline 5 & 6 & 13 & 12 & 9 \\
\hline 5 & 6 & 13 & 45 & 9 \\
\hline 5 & 6 & 13 & 14 & 9 \\
\hline 7 & 6 & 13 & 12 & 9 \\
\hline 7 & 6 & 13 & 45 & 9 \\
\hline 7 & 6 & 13 & 14 & \\
\hline 8 & 6 & 13 & 12 & 9 \\
\hline 8 & 6 & 13 & 45 & 9 \\
\hline 8 & 6 & 13 & 14 & \\
\hline 13 & 6 & 7 & 34 & \\
\hline 13 & 6 & 7 & 35 & \\
\hline 13 & 6 & 7 & 36 & o \\
\hline
\end{tabular}




\begin{tabular}{|c|c|c|c|c|}
\hline 5 & 6 & 73 & 34 & 9 \\
\hline 5 & 6 & 7 & 35 & 9 \\
\hline 5 & 6 & 7 & 36 & 9 \\
\hline 8 & 6 & 73 & 34 & 9 \\
\hline 8 & 6 & 73 & 35 & 9 \\
\hline 8 & 6 & 73 & 36 & 9 \\
\hline 13 & 6 & 8 & 9 & 9 \\
\hline 13 & 6 & 8 & 37 & 9 \\
\hline 13 & 6 & 8 & 38 & 9 \\
\hline 5 & 6 & 8 & 9 & 9 \\
\hline 5 & 6 & 8 & 37 & 9 \\
\hline 5 & 6 & 8 & 38 & 9 \\
\hline 7 & 6 & 8 & 9 & 9 \\
\hline 7 & 6 & 8 & 37 & 9 \\
\hline 7 & 6 & 8 & 38 & 9 \\
\hline 37 & 8 & 9 & 10 & 9 \\
\hline 37 & 8 & 9 & 40 & 9 \\
\hline 37 & 8 & 9 & 39 & 9 \\
\hline 6 & 8 & 9 & 10 & 9 \\
\hline 6 & 8 & 9 & 40 & 9 \\
\hline 6 & 8 & 93 & 39 & 9 \\
\hline 38 & 8 & 9 & 10 & 9 \\
\hline 38 & 8 & 9 & 40 & 9 \\
\hline 38 & 8 & 9 & 39 & 9 \\
\hline 40 & 9 & 10 & 41 & 9 \\
\hline 40 & 9 & 10 & 11 & 9 \\
\hline 40 & 9 & 10 & 12 & 9 \\
\hline 39 & 9 & 10 & 41 & 9 \\
\hline 39 & 9 & 10 & 11 & 9 \\
\hline 39 & 9 & 10 & 12 & 9 \\
\hline 8 & 9 & 10 & 41 & 9 \\
\hline 8 & 9 & 10 & 11 & 9 \\
\hline 8 & 9 & 10 & 12 & 9 \\
\hline 9 & 10 & 11 & 42 & 9 \\
\hline 41 & 10 & 11 & 42 & 9 \\
\hline 12 & 10 & 11 & 42 & 9 \\
\hline 9 & 10 & 12 & 43 & 9 \\
\hline 9 & 10 & 12 & 44 & 9 \\
\hline 9 & 10 & 12 & 13 & 9 \\
\hline 41 & 10 & 12 & 43 & 9 \\
\hline 41 & 10 & 12 & 44 & 9 \\
\hline 41 & 10 & 12 & 13 & 9 \\
\hline 11 & 10 & 12 & 43 & 9 \\
\hline 11 & 10 & 12 & 44 & 9 \\
\hline 11 & 10 & 12 & 13 & 9 \\
\hline 10 & 12 & 13 & 45 & 9 \\
\hline
\end{tabular}




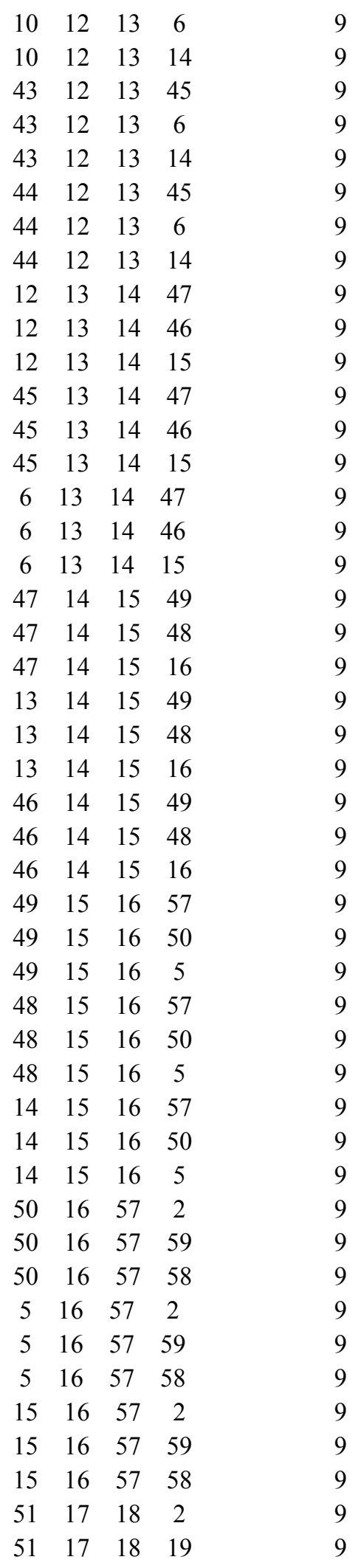




\begin{tabular}{|c|c|c|c|c|}
\hline 51 & 17 & 18 & 53 & 9 \\
\hline 52 & 17 & 18 & 2 & 9 \\
\hline 52 & 17 & 18 & 19 & 9 \\
\hline 52 & 17 & 18 & 53 & 9 \\
\hline 58 & 17 & 18 & 2 & 9 \\
\hline 58 & 17 & 18 & 19 & 9 \\
\hline 58 & 17 & 18 & 53 & 9 \\
\hline 18 & 17 & 52 & 61 & 9 \\
\hline 51 & 17 & 52 & 61 & 9 \\
\hline 58 & 17 & 52 & 61 & 9 \\
\hline 18 & 17 & 58 & 57 & 9 \\
\hline 18 & 17 & 58 & 59 & 9 \\
\hline 18 & 17 & 58 & 60 & 9 \\
\hline 51 & 17 & 58 & 57 & 9 \\
\hline 51 & 17 & 58 & 59 & 9 \\
\hline 51 & 17 & 58 & 60 & 9 \\
\hline 52 & 17 & 58 & 57 & 9 \\
\hline 52 & 17 & 58 & 59 & 9 \\
\hline 52 & 17 & 58 & 60 & 9 \\
\hline 17 & 18 & 19 & 25 & 9 \\
\hline 17 & 18 & 19 & 20 & 9 \\
\hline 2 & 18 & 19 & 25 & 9 \\
\hline 2 & 18 & 19 & 20 & 9 \\
\hline 53 & 18 & 19 & 25 & 9 \\
\hline 53 & 18 & 19 & 20 & 9 \\
\hline 18 & 19 & 25 & 56 & 9 \\
\hline 18 & 19 & 25 & 24 & 9 \\
\hline 20 & 19 & 25 & 56 & 9 \\
\hline 20 & 19 & 25 & 24 & 9 \\
\hline 25 & 19 & 20 & 21 & 9 \\
\hline 25 & 19 & 20 & 54 & 9 \\
\hline 18 & 19 & 20 & 21 & 9 \\
\hline 18 & 19 & 20 & 54 & 9 \\
\hline 19 & 20 & 21 & 22 & 9 \\
\hline 19 & 20 & 21 & 55 & 9 \\
\hline 54 & 20 & 21 & 22 & 9 \\
\hline 54 & 20 & 21 & 55 & 9 \\
\hline 20 & 21 & 22 & 23 & 9 \\
\hline 20 & 21 & 22 & 24 & 9 \\
\hline 55 & 21 & 22 & 23 & 9 \\
\hline 55 & 21 & 22 & 24 & 9 \\
\hline 21 & 22 & 24 & 25 & 9 \\
\hline 23 & 22 & 24 & 25 & 9 \\
\hline 22 & 24 & 25 & 19 & 9 \\
\hline 22 & 24 & 25 & 56 & 9 \\
\hline 17 & 52 & 61 & 62 & \\
\hline
\end{tabular}




$\begin{array}{ccccc}17 & 52 & 61 & 63 & 9 \\ 2 & 57 & 59 & 58 & 9 \\ 16 & 57 & 59 & 58 & 9 \\ 2 & 57 & 58 & 17 & 9 \\ 2 & 57 & 58 & 59 & 9 \\ 2 & 57 & 58 & 60 & 9 \\ 59 & 57 & 58 & 17 & 9 \\ 59 & 57 & 58 & 60 & 9 \\ 16 & 57 & 58 & 17 & 9 \\ 16 & 57 & 58 & 59 & 9 \\ 16 & 57 & 58 & 60 & 9 \\ 17 & 58 & 59 & 57 & 9 \\ 60 & 58 & 59 & 57 & 9 \\ 52 & 61 & 62 & 65 & 9 \\ 52 & 61 & 62 & 66 & 9 \\ 52 & 61 & 62 & 64 & 9 \\ 63 & 61 & 62 & 65 & 9 \\ 63 & 61 & 62 & 66 & 9 \\ 63 & 61 & 62 & 64 & 9\end{array}$

[ dihedrals ]

; ai aj ak al funct $\mathrm{c} 0 \mathrm{c} 1 \mathrm{c} 2 \mathrm{c} 3$

$\begin{array}{llllll}22 & 21 & 23 & 24 & 2\end{array}$

$\begin{array}{lllll}61 & 62 & 63 & 52 & 2\end{array}$ 
Supporting data file S4. Cinobufagin prm file.

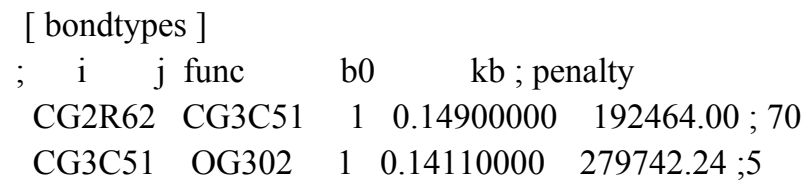




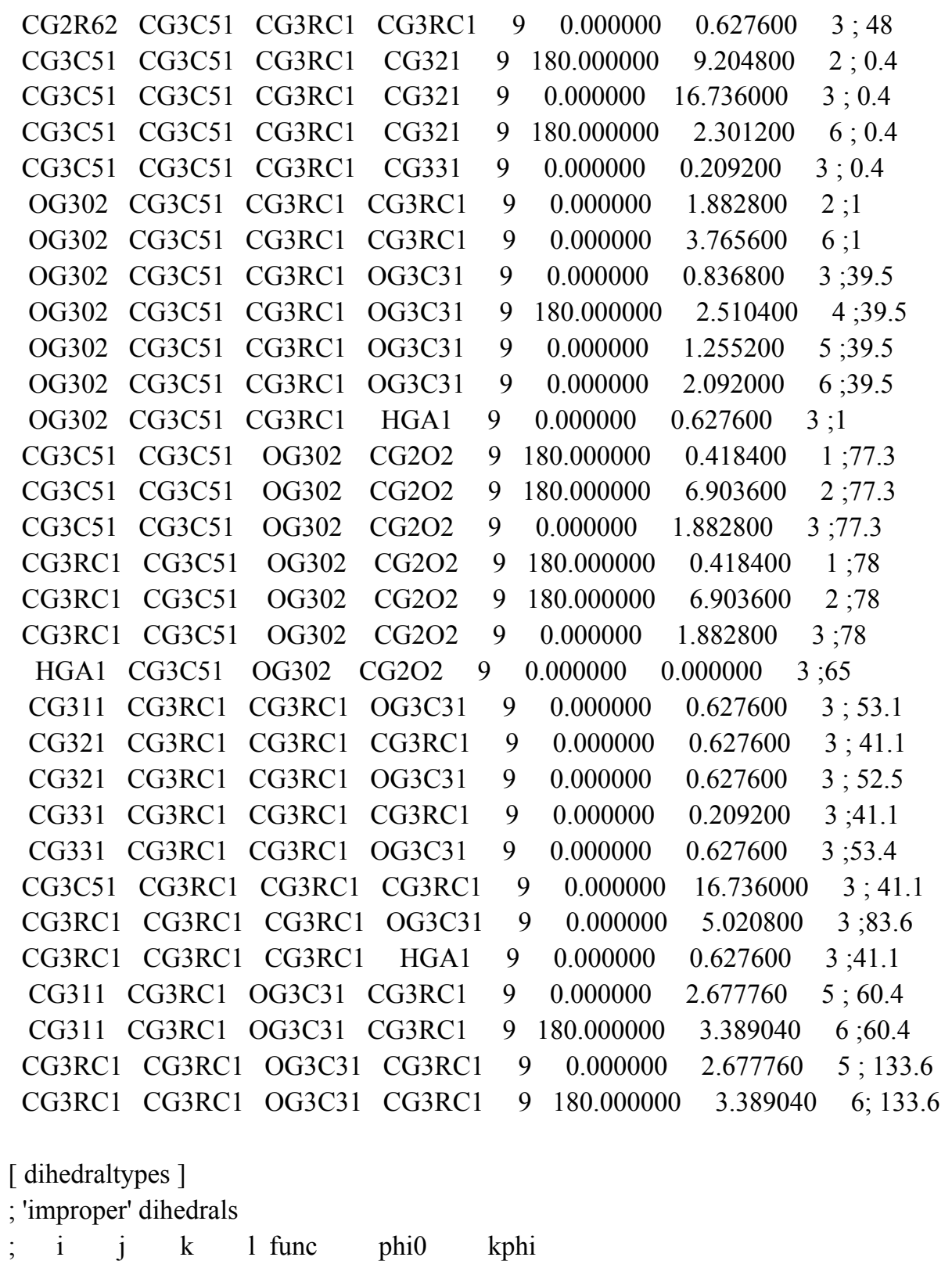

